\title{
Some geometrical methods for constructing contradiction measures on Atanassov's intuitionistic fuzzy sets
}

\author{
Elena E. Castiñeira ${ }^{1}$, Carmen Torres-Blanc* and Susana Cubillo ${ }^{2}$ \\ Department of Applied Mathematics, Technical University of Madrid (UPM), 28660 Boadilla del \\ Monte, Madrid, Spain
}

\begin{abstract}
Trillas et al. (1999, Soft computing, 3 (4), 197-199) and Trillas and Cubillo (1999, On non-contradictory input/output couples in Zadeh's CRI proceeding, 28-32) introduced the study of contradiction in the framework of fuzzy logic because of the significance of avoiding contradictory outputs in inference processes. Later, the study of contradiction in the framework of Atanassov's intuitionistic fuzzy sets (A-IFSs) was initiated by Cubillo and Castiñeira (2004, Contradiction in intuitionistic fuzzy sets proceeding, 2180-2186). The axiomatic definition of contradiction measure was stated in Castiñeira and Cubillo (2009, International journal of intelligent systems, 24, 863-888). Likewise, the concept of continuity of these measures was formalized through several axioms. To be precise, they defined continuity when the sets 'are increasing', denominated continuity from below, and continuity when the sets 'are decreasing', or continuity from above. The aim of this paper is to provide some geometrical construction methods for obtaining contradiction measures in the framework of A-IFSs and to study what continuity properties these measures satisfy. Furthermore, we show the geometrical interpretations motivating the measures.
\end{abstract}

Keywords: Atanassov's intuitionistic fuzzy sets; contradiction measures; semicontinuous measures; semilattices; order isomorphism and automorphism

\section{Introduction}

Due to the significance of avoiding contradictory outputs in inference processes, Trillas et al. (1999) and Trillas and Cubillo (1999) studied contradiction in the framework of fuzzy logic by introducing the concept of the contradictory set. They established that the fuzzy set associated with the predicate $P$, and determined by the membership function $\mu_{P}$, is contradictory if ' $\mu_{P}(x) \rightarrow \mu_{\neg P}(x)$ for all $x$ ', representing the implication ' $\rightarrow$ ' by means of the reticular order $\leq$ of $[0,1]$ and $\neg P$ through some strong negation $N$. Remember that $N:[0,1] \rightarrow[0,1]$ is a strong fuzzy negation if it is a decreasing function satisfying $N(0)=1, N(1)=0$, and $N^{2}=i d$. Thus, $\mu_{P}$ is said to be contradictory regarding a strong negation $N$, or $N$-contradictory, if $\mu_{P} \leq N \circ \mu_{P}$. This definition was generalized by Trillas et al. (2002) with the aim of studying the laws of non-contradiction and excluded middle from a new point of view. In that paper, the implication ' $\rightarrow$ ' is replaced by a transitive relation ' $\vDash$ ' and the negation ' $\neg$ ' by a unary operation that is $\vDash$-reversing.

In classical logic, the inequality $\mu_{P} \leq N \circ \mu_{P}$ is true if and only if $\mu_{P}=\mu_{\emptyset}$. This would lead to an alternative interpretation of the inequality $\mu_{P} \leq N \circ \mu_{P}$, namely, that $P$ is

*Corresponding author. Email: ctorres@fi.upm.es 
'weakly empty' with respect to $N$. In this sense, Piasecki (1985) established that a set $P$ is $w$-empty if $\mu_{P} \leq 1-\mu_{P}$.

Nevertheless, the extent to which the condition $\mu_{P} \leq N \circ \mu_{P}$ holds, that is, how contradictory $\mu_{P}$ is, is a matter for consideration, since $\mu_{P}$ can behave quite differently regarding this characteristic. The need to speak not only of contradiction but also of degrees of contradiction was later raised by Castiñeira et al. (2002), where some functions were considered for the purpose of determining that degree. Later, an axiomatic model for measuring how contradictory a fuzzy set is was proposed by Cubillo and Castiñeira (2005). Then, an axiomatic model to measure the contradiction in Atanassov's intuitionistic fuzzy sets (A-IFSs) was established by Castiñeira and Cubillo (2009).

In this paper, we devise several methods for constructing contradiction measures on A-IFSs. The paper is organized as follows. Section 2 establishes everything we need to know about Atanassov's fuzzy sets and contradiction measures to explain the new results. Section 3 deals with contradiction measures that are completely semicontinuous. Finally, Section 4 introduces some semicontinuous contradiction measures. In all cases, after studying what kind of continuity the measures satisfy, we present the geometrical interpretations that really motivated the construction of such measures.

\section{Preliminaries}

\subsection{On the Atanassov intuitionistic fuzzy sets}

Given a fuzzy predicate $\mathbf{A}$ in a universe of discourse $X \neq \emptyset$, an A-IFS associated with $\mathbf{A}$ (see Atanassov 1999) is a set $A=\left\{\left(x, \mu_{A}(x), \nu_{A}(x)\right): x \in X\right\}$, where $\mu_{A}: X \rightarrow[0,1]$, $\nu_{A}: X \rightarrow[0,1]$ are called, respectively, the membership and non-membership functions, and such that, for all $x \in X, \mu_{A}(x)+\nu_{A}(x) \leq 1$. Let us denote the set of all A-IFSs on $X$ as $\mathcal{I} \mathcal{F}(X)$.

The A-IFSs could be considered as what Goguen termed $L$-fuzzy sets (Goguen 1967), where the lattice $L$ is the set $\mathbb{L}=\left\{\left(\alpha_{1}, \alpha_{2}\right) \in[0,1]^{2}: \alpha_{1}+\alpha_{2} \leq 1\right\}$ with the partial order $\leq_{\mathbb{L}}$ defined as follows: given $\boldsymbol{\alpha}=\left(\alpha_{1}, \alpha_{2}\right), \boldsymbol{\beta}=\left(\beta_{1}, \beta_{2}\right) \in \mathbb{L}, \boldsymbol{\alpha} \leq_{\mathbb{L}} \boldsymbol{\beta}$ holds if and only if $\alpha_{1} \leq \beta_{1}$ and $\alpha_{2} \geq \beta_{2}$. ( $\left(\mathbb{L}, \leq_{\mathbb{L}}\right)$ is a complete lattice with least element, $0_{\mathbb{L}}=(0,1)$, and greatest element, $1_{\mathbb{L}}=(1,0)$.

So, an A-IFS $A$ is an $\mathbb{L}$-fuzzy set whose $\mathbb{L}$-membership function $\chi^{A} \in \mathbb{L}^{X}=\{\chi$ : $X \rightarrow \mathbb{L}\}$ is defined for each $x \in X$ as $\chi^{A}(x)=\left(\mu_{A}(x), \nu_{A}(x)\right)$.

The order $\leq_{\mathbb{L}}$ of $\mathbb{L}$ naturally induces a partial order on $\mathbb{L}^{X}$, which is given as follows: if $\chi^{A}, \chi^{B} \in \mathbb{L}^{X}, \chi^{A} \leq_{\mathbb{L}} \chi^{B}$ holds if and only if $\chi^{A}(x) \leq_{\mathbb{L}} \chi^{B}(x)$ for all $x \in X$. Thus, $\left(\mathbb{L}^{X}, \leq_{\mathbb{L}}\right)$ is a bounded and complete lattice in which the least and greatest elements are, respectively, $\chi^{0_{\mathfrak{L}}}$ and $\chi^{1_{\mathfrak{L}}}$ defined by $\chi^{0_{\mathfrak{L}}}(x)=0_{\mathbb{L}}$ and $\chi^{1_{\mathfrak{L}}}(x)=1_{\mathbb{L}}$ for all $x \in X$.

Furthermore, let us recall that a decreasing function $\mathcal{N}: \mathbb{L} \rightarrow \mathbb{L}$ is an intuitionistic fuzzy negation (IFN) if $\mathcal{N}\left(0_{\mathbb{L}}\right)=1_{\mathbb{R}}$ and $\mathcal{N}\left(1_{\mathbb{L}}\right)=0_{\mathbb{L}}$ hold; and $\mathcal{N}$ is a strong IFN if the equality $\mathcal{N}(\mathcal{N}(\boldsymbol{\alpha}))=\boldsymbol{\alpha}$ holds for all $\boldsymbol{\alpha} \in \mathbb{L}$ (for more about IFN, see Bustince et al. 2000, Deschrijver et al. 2002, 2004).

\subsection{On the contradiction on A-IFSs}

The study of contradiction in the framework of A-IFSs was initiated by Cubillo and Castiñeira (2004). As in the fuzzy case, an A-IFS $A$, or alternatively $\chi^{A} \in \mathbb{L}^{X}$, is said to be contradictory with respect to some strong IFN $\mathcal{N}$, or $A$ is $\mathcal{N}$-contradictory, if $\chi^{A}(x) \leq_{\mathbb{L}}$ $\left(\mathcal{N} \circ \chi^{A}\right)(x)$ for all $x \in X$, where $\chi^{A}$ is the $\mathbb{L}$-membership function of $A$. Also, $A$ (or $\chi^{A}$ ) is said to be contradictory if there exists a strong negation $\mathcal{N}$ such that $A$ is $\mathcal{N}$-contradictory. 
As we want to know not only whether a set is contradictory, but also to what extent this property holds, we dealt with the problem of measuring the contradiction in the case of A-IFSs. A general and axiomatic model for measuring contradiction on $\mathcal{I} \mathcal{F}(X)$ was presented by Castiñeira and Cubillo (2009). They established and justified a number of axioms; moreover, they gave several examples to illustrate those new concepts. The first definition presented there is as follows.

DEFINITION 2.1 (CASTIÑEIRA AND Cubillo 2009). Let $X \neq \emptyset$ be a universe of discourse, a function $\mathcal{C}: \mathbb{L}^{X} \rightarrow[0,1]$ is a measure of contradiction on the $\mathcal{I} \mathcal{F}(X)$, or on $\mathbb{L}^{X}$, if the following is satisfied:

(c.i) $\mathcal{C}\left(\chi^{0_{\Perp}}\right)=1$;

(c.ii) if $\chi=(\mu, \nu) \in \mathbb{L}^{X}$ satisfies $\operatorname{Inf}_{x \in X} \nu(x)=0$ (we say that $\chi$ is $\mathbb{L}$-normal), then $\mathcal{C}(\chi)=0$

(c.iii) anti-monotonicity: given $\chi^{A}, \chi^{B} \in \mathbb{L}^{X}$ such that $\chi^{A} \leq_{\mathbb{L}} \chi^{B}$, then $\mathcal{C}\left(\chi^{A}\right) \geq \mathcal{C}\left(\chi^{B}\right)$.

The set of all measures of contradiction on $\mathbb{\complement}^{X}$ was denoted by $\mathcal{C M}(\mathcal{I} \mathcal{F}(X))$ or more concisely $\mathcal{C M}\left(\mathbb{L}^{X}\right)$.

Furthermore, as the above definition does not determine gradually varying degrees of contradiction, other axioms were also introduced to model the continuity, from both below and above, of contradiction measures, as follows.

DEFINITION 2.2 (CASTIÑEIRA AND CubILlo 2009). Let $X \neq \emptyset$, a contradiction measure $\mathcal{C}: \mathbb{L}^{X} \rightarrow[0,1]$ is said to be

- completely semicontinuous from below if the following axiom is satisfied:

(c.iv) for each indexed family $\left\{\chi^{i}\right\}_{i \in \mathcal{I}} \subset \mathbb{L}^{X}$,

$$
\operatorname{Inf}_{i \in \mathcal{I}} \mathcal{C}\left(\chi^{i}\right)=\mathcal{C}\left(\operatorname{Sup}_{i \in \mathcal{I}} \chi^{i}\right)
$$

holds, where $\operatorname{Sup}_{i \in \mathcal{I}} \chi^{i} \in \mathbb{L}^{X}$ is defined for all $x \in X$ as $\left(\operatorname{Sup}_{i \in \mathcal{I}} \chi^{i}\right)(x)=$ $\operatorname{Sup}_{i \in \mathcal{I}} \chi^{i}(x)$;

- completely semicontinuous from above if the following axiom is satisfied:

(c.v) for each indexed family $\left\{\chi^{i}\right\}_{i \in \mathcal{I}} \subset \mathbb{L}^{X} \backslash \mathbb{L}_{0}^{X}$, where $\mathbb{L}_{0}^{X}=\left\{\chi \in \mathbb{L}^{X}\right.$ : $\chi$ is $\mathbb{L}$-normal\},

$$
\operatorname{Sup}_{i \in \mathcal{I}} \mathcal{C}\left(\chi^{i}\right)=\mathcal{C}\left(\operatorname{Inf}_{i \in \mathcal{I}} \chi^{i}\right)
$$

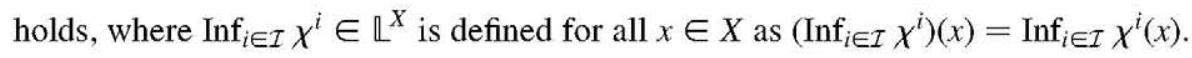

Remark 1 . Note that for each $x \in X$, we have the A-IFS on $\mathcal{I}$ defined by

$$
A_{x}=\left\{\left(i, \mu_{i}(x), \nu_{i}(x)\right): i \in \mathcal{I}\right\},
$$

whose closure (Atanassov 1986) is the A-IFS

$$
C\left(A_{x}\right)=\left\{\left(i, \operatorname{Sup}_{i \in \mathcal{I}} \mu_{i}(x), \operatorname{Inf}_{i \in \mathcal{I}} \nu_{i}(x)\right): i \in \mathcal{I}\right\}
$$


which, considered as an $\mathbb{L}$-fuzzy set on $\mathcal{I}$, has the $\mathbb{L}$-membership function

$$
\operatorname{Sup}_{i \in \mathcal{I}} \chi^{i}(x)=\left(\operatorname{Sup}_{i \in \mathcal{I}} \mu_{i}(x), \operatorname{Inf}_{i \in \mathcal{I}} \nu_{i}(x)\right) .
$$

Note also that for each $x \in X$, the interior (Atanassov 1986) of $A_{x}$ is the A-IFS

$$
I\left(A_{x}\right)=\left\{\left(i, \operatorname{Inf}_{i \in \mathcal{I}} \mu_{i}(x), \operatorname{Sup}_{i \in \mathcal{I}} \nu_{i}(x)\right): i \in \mathcal{I}\right\},
$$

which, considered as an $\mathbb{L}$-fuzzy set on $\mathcal{I}$, has the $\mathbb{L}$-membership function

$$
\operatorname{Inf}_{i \in \mathcal{I}} \chi^{i}(x)=\left(\operatorname{Inf}_{i \in \mathcal{I}} \mu_{i}(x), \operatorname{Sup}_{i \in \mathcal{I}} \nu_{i}(x)\right) .
$$

The set of all contradiction measures that are completely semicontinuous from below on $\mathcal{I} \mathcal{F}(X)$ was denoted by $\mathcal{C} \mathcal{M}_{c s c}\left(\mathbb{L}^{X}\right)$ and the set of all contradiction measures that are completely semicontinuous from above by $\mathcal{C} \mathcal{M}^{c s c}\left(\mathbb{L}^{X}\right)$.

Remark 2. In the above paper, Castiñeira and Cubillo (2009), it was proved that the functions $\mathcal{C}_{*}, \mathcal{C}^{*}: \mathbb{L}^{X} \rightarrow[0,1]$ defined, respectively, for each $\chi=(\mu, \nu) \in \mathbb{L}^{X}$ as

$$
\mathcal{C}_{*}(\chi)=\operatorname{Inf}_{x \in X} \nu(x) \text { and } \mathcal{C}^{*}(\chi)= \begin{cases}0 & \text { if } \chi \in \mathbb{L}_{0}^{X} \\ \operatorname{Sup}_{x \in X} \nu(x) & \text { if } \chi \in \mathbb{L}^{X} \backslash \mathbb{L}_{0}^{X}\end{cases}
$$

satisfy $\mathcal{C}_{*} \in \mathcal{C} \mathcal{M}_{c s c}\left(\mathbb{L}^{X}\right)$ and $\mathcal{C}^{*} \in \mathcal{C} \mathcal{M}^{c s c}\left(\mathbb{L}^{X}\right)$.

Nevertheless, the axioms (c.iv) and (c.v) of complete continuity could appear to be too restrictive because there exist contradiction measures, in which the values change gradually, like the functions proposed by Castiñeira et al. (2006), which do not satisfy them. For this reason, we established other weaker axioms using semilattices. Before we state these axioms, remember that [see Blyth (2005) or Birkhoff (1940)] a set $S \subset \mathbb{L}^{X}$ is an upper semilattice if for all $\chi^{A}, \chi^{B} \in S$, $\operatorname{Sup}\left\{\chi^{A}, \chi^{B}\right\} \in S$ holds; and $S \subset \mathbb{L}^{X}$ is a lower semilattice if for all $\chi^{A}, \chi^{B} \in S, \operatorname{Inf}\left\{\chi^{A}, \chi^{B}\right\} \in S$ holds.

DeFINITION 2.3 (CASTIÑEIRA AND Cubillo 2009). Let $X \neq \emptyset$, a contradiction measure $\mathcal{C}: \mathbb{L}^{X} \rightarrow[0,1]$ is said to be

- semicontinuous from below if it satisfies the following axiom:

(c.vi) for each upper semilattice $\left\{\chi^{i}\right\}_{i \in \mathcal{I}} \subset \mathbb{L}^{X}$, the following holds:

$$
\operatorname{Inf}_{i \in \mathcal{I}} \mathcal{C}\left(\chi^{i}\right)=\mathcal{C}\left(\operatorname{Sup}_{i \in \mathcal{I}} \chi^{i}\right)
$$

- semicontinuous from above if it satisfies the following axiom:

(c.vii) for each lower semilattice $\left\{\chi^{i}\right\}_{i \in \mathcal{I}} \subset \mathbb{L}^{X} \backslash \mathbb{L}_{0}^{X}$, the following holds:

$$
\operatorname{Sup}_{i \in \mathcal{I}} \mathcal{C}\left(\chi^{i}\right)=\mathcal{C}\left(\operatorname{Inf}_{i \in \mathcal{I}} \chi^{i}\right) \text {. }
$$


The set of all contradiction measures that are semicontinuous from below on $\mathbb{L}^{X}$ was denoted by $\mathcal{C M}_{s c}\left(\mathbb{L}^{X}\right)$ and the set of all contradiction measures that are semicontinuous from above by $\mathcal{C} \mathcal{M}^{s c}\left(\mathbb{L}^{X}\right)$.

Moreover, it was shown by Castiñeira and Cubillo (2009) that

$$
\begin{aligned}
& \emptyset \neq \mathcal{C M}_{c s c}\left(\mathbb{L}^{X}\right) \subsetneq \mathcal{C} \mathcal{M}_{s c}\left(\mathbb{L}^{X}\right) \subsetneq \mathcal{C M}\left(\mathbb{L}^{X}\right), \\
& \emptyset \neq \mathcal{C M}^{c s c}\left(\mathbb{L}^{X}\right) \subsetneq \mathcal{C} \mathcal{M}^{s c}\left(\mathbb{L}^{X}\right) \subsetneq \mathcal{C M}\left(\mathbb{L}^{X}\right) .
\end{aligned}
$$

Finally, it is important to take into account that

- every axiom of continuity implies anti-monotonicity;

- if $\chi^{i}=\left(\mu_{i}, \nu_{i}\right)$ for all $i \in \mathcal{I}$, then $\operatorname{Inf}_{i \in \mathcal{I}} \chi^{i}=\left(\operatorname{Inf}_{i \in \mathcal{I}} \mu_{i}, \operatorname{Sup}_{i \in \mathcal{I}} \nu_{i}\right)$ and $\operatorname{Sup}_{i \in \mathcal{I}} \chi^{i}=\left(\operatorname{Sup}_{i \in \mathcal{I}} \mu_{i}, \operatorname{Inf}_{i \in \mathcal{I}} \nu_{i}\right)$.

\section{Families of completely semicontinuous contradiction measures}

In this section, we introduce three families of functions showing that they are contradiction measures that are completely semicontinuous from below. Also, we construct other functions or contradiction measures that are completely semicontinuous from above. In all cases, we study not only what kind of continuity the functions satisfy but also what they do not. We also state the geometrical interpretations that have suggested the construction of those functions.

THEOREM 3.1. If $\varphi:[0,1] \rightarrow[0,1]$ is an order automorphism, then the following is satisfied.

(a) The function $\mathcal{C}_{\varphi, *}: \mathbb{L}^{X} \rightarrow[0,1]$ defined for each $\chi=(\mu, \nu) \in \mathbb{L}^{X}$ by $\mathcal{C}_{\varphi, *}(\chi)=$ $\operatorname{Inf}_{x \in X}\left(\varphi^{\circ} \nu\right)(x)$ is a contradiction measure that is completely semicontinuous from below.

(b) The function $\mathcal{C}^{\varphi, *}: \mathbb{L}^{X} \rightarrow[0,1]$ defined by

$$
\mathcal{C}^{\varphi, *}(\chi)= \begin{cases}0 & \text { if } \chi \in \mathbb{L}_{0}^{X} \\ \operatorname{Sup}_{x \in X}(\varphi \circ \nu)(x) & \text { if } \chi \in \mathbb{L}^{X} \backslash \mathbb{L}_{0}^{X}\end{cases}
$$

is a contradiction measure that is completely semicontinuous from above.

Proof. All the axioms of contradiction measures and their continuity are trivially preserved under any order automorphism. Thus, it follows from $\mathcal{C}_{\varphi, *}=\varphi^{\circ} \mathcal{C}_{*}$ and $\mathcal{C}^{\varphi, *}=\varphi^{\circ} \mathcal{C}^{*}$, where $\mathcal{C}_{*}$ and $\mathcal{C}^{*}$ are the contradiction measures presented in Remark 2, that $\mathcal{C}_{\varphi, *} \in$ $\mathcal{C} \mathcal{M}_{c s c}\left(\mathbb{L}^{X}\right)$ and $\mathcal{C}^{\varphi, *} \in \mathcal{C} \mathcal{M}^{c s c}\left(\mathbb{L}^{X}\right)$.

Figure 1 shows a simple geometrical interpretation of the measures $\mathcal{C}_{\varphi, *}$ and $\mathcal{C}^{\varphi, *}$.

Example 3.2. Let $\left\{N_{\lambda}\right\}_{\lambda \in(-1, \infty)}$ be the family of Sugeno's negations (Sugeno 1974), defined for all $\alpha \in[0,1]$ by $N_{\lambda}(\alpha)=(1-\alpha) /(1+\lambda \alpha)$; and let $\left\{N_{\gamma}\right\}_{\gamma \in(0, \infty)}$ be the family of Yager's negations (Yager 1980), defined for all $\alpha \in[0,1]$ by $N_{\gamma}(\alpha)=\left(1-\alpha^{\gamma}\right)^{1 / \gamma}$. Then, 


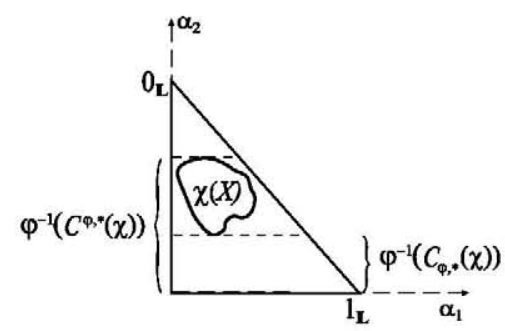

Figure 1. Geometrical interpretation of the measures $\mathcal{C}_{\varphi, *}$ and $\mathcal{C}^{\varphi, *}$.

(a) if $\varphi_{\lambda}=N_{\lambda} \circ N_{s}$, where $N_{s}$ is the standard negation $N_{s}(\alpha)=1-\alpha$, then $\mathcal{C}_{\varphi_{\lambda}, *}(\chi)=\operatorname{Inf}_{x \in X}(\nu(x) /(1+\lambda-\lambda \nu(x)))$, for each $\chi=(\mu, \nu) \in \mathbb{L}^{X}$, defines a contradiction measure that is completely semicontinuous from below on $\mathbb{L}^{X}$; analogously, the function defined for each $\chi=(\mu, \nu) \in \mathbb{L}^{X} \backslash \mathbb{L}_{0}^{X}$ by $\mathcal{C}^{\varphi_{\lambda}, *}(\chi)=$ $\operatorname{Sup}_{x \in X}(\nu(x) /(1+\lambda-\lambda \nu(x)))$ and $\mathcal{C}^{\varphi_{\lambda}, *}(\chi)=0$ if $\chi \in \mathbb{L}_{0}^{X}$ satisfies $\mathcal{C}^{\varphi_{\lambda}, *} \in \mathcal{C M}^{c s c}$ $\left(\mathbb{L}^{X}\right)$;

(b) if $\varphi_{\gamma}=N_{\gamma} \circ N_{s}$, then $\mathcal{C}_{\varphi_{\gamma}, *}(\chi)=\operatorname{Inf}_{x \in X}\left(1-(1-\nu(x))^{\gamma}\right)^{1 / \gamma}$, for each $\chi \in \mathbb{L}^{X}$, defines a contradiction measure that is completely semicontinuous from below on $\mathbb{L}^{X}$, whereas $\mathcal{C}^{\varphi_{\gamma}, *}(\chi)=\operatorname{Sup}_{x \in X}\left(1-(1-\nu(x))^{\gamma}\right)^{1 / \gamma}$ if $\chi \in \mathbb{L}^{X} \backslash \mathbb{L}_{0}^{X}$, and $\mathcal{C}^{\varphi_{\gamma}, *}(\chi)=0$ if $\chi \in \mathbb{L}_{0}^{X}$, defines a contradiction measure that is completely semicontinuous from above.

We know that the measures $\mathcal{C}_{\varphi, *}$ and $\mathcal{C}^{\varphi, *}$ satisfy $\mathcal{C}_{\varphi, *} \in \mathcal{C} \mathcal{M}_{c s c}\left(\mathbb{L}^{X}\right) \subset \mathcal{C} \mathcal{M}_{s c}\left(\mathbb{L}^{X}\right)$ and $\mathcal{C}^{\varphi, *} \in \mathcal{C} \mathcal{M}^{c s c}\left(\mathbb{L}^{X}\right) \subset \mathcal{C} \mathcal{M}^{s c}\left(\mathbb{L}^{X}\right)$. Now let us see what kind of continuity they do not satisfy.

Proposition 3.3. Let $\varphi:[0,1] \rightarrow[0,1]$ be an order automorphism and let $X$ be an infinite set, then the following is satisfied.

(a) $\mathcal{C}_{\varphi, *} \notin \mathcal{C} \mathcal{M}^{s c}\left(\mathbb{L}^{X}\right)$ and consequently $\mathcal{C}_{\varphi, *} \notin \mathcal{C} \mathcal{M}^{c s c}\left(\mathbb{L}^{X}\right)$.

(b) $\mathcal{C}^{\varphi, *} \notin \mathcal{C} \mathcal{M}_{s c}\left(\mathbb{L}^{X}\right)$ and consequently $\mathcal{C}^{\varphi, *} \notin \mathcal{C} \mathcal{M}_{c s c}\left(\mathbb{L}^{X}\right)$.

Proof. We are going to prove only (a) because the proof of (b) is similar. Let $\mathcal{P}_{F}(X)$ be the family of all finite subsets of $X$. We consider the family of A-IFSs taking the same value $0_{\mathbb{L}}$ on a finite number of elements of the universe, and the value $(0,1 / 2)$ on the others, i.e. $\left\{\chi^{A}\right\}_{A \in \mathcal{P}_{F}(X)} \subset \mathbb{L}^{X} \backslash \mathbb{L}_{0}^{X}$ such that for each $A \in \mathcal{P}_{F}(X)$,

$$
\chi^{A}(x)= \begin{cases}0_{\mathbb{L}} & \text { if } x \in A \\ \left(0, \frac{1}{2}\right) & \text { if } x \notin A .\end{cases}
$$

We have that $\left\{\chi^{A}\right\}_{A \in \mathcal{P}_{F}(X)} \subset \mathbb{L}^{X} \backslash \mathbb{L}_{0}^{X}$ is a lower semilattice. Indeed, if $\chi^{A_{1}}, \chi^{A_{2}} \in$ $\left\{\chi^{A}\right\}_{A \in \mathcal{P}_{F}(X)}$, then $\operatorname{Inf}\left\{\chi^{A_{1}}, \chi^{A_{2}}\right\}=\chi^{A_{1} \cup A_{2}}$, where $\chi^{A_{1} \cup A_{2}} \in\left\{\chi^{A}\right\}_{A \in \mathcal{P}_{F}(X)}$ as $A_{1} \cup A_{2}$ is a finite set.

Thus, $\mathcal{C}_{\varphi, *}\left(\chi^{A}\right)=\varphi(1 / 2) \neq 1$ for all $\chi^{A} \in\left\{\chi^{A}\right\}_{A \in \mathcal{P}_{F}(X)}$ and so

$$
\operatorname{Sup}_{A \in \mathcal{P}_{F}(X)} \mathcal{C}_{\varphi, *}\left(\chi^{A}\right)=\varphi\left(\frac{1}{2}\right) \neq 1 .
$$

However, $\mathcal{C}_{\varphi, *}\left(\operatorname{Inf}_{A \in \mathcal{P}_{F}(X)} \chi^{A}\right)=\mathcal{C}_{\varphi, *}\left(\chi^{0_{\mathfrak{L}}}\right)=1$. Therefore, $\mathcal{C}_{\varphi, *} \notin \mathcal{C} \mathcal{M}^{s c}\left(\mathbb{L}^{X}\right)$. 
The following results show contradiction measures taking discrete values in $[0,1]$. They are general constructions based on two cases given by Castiñeira and Cubillo (2009). Therefore, we omit their proofs because they are similar to the proofs of those cases.

To simplify the notation, we consider intervals in $\mathbb{L}$ with some extreme in $[0,1]^{2}$ as follows: if $\beta=\left(\beta_{1}, \beta_{2}\right) \in[0,1]^{2}$,

$$
\begin{aligned}
& {\left[0_{\mathbb{L}}, \boldsymbol{\beta}\right]=\left\{\left(\alpha_{1}, \alpha_{2}\right) \in \mathbb{L}: \alpha_{1} \leq \beta_{1} \& \alpha_{2} \geq \beta_{2}\right\},} \\
& {\left[\boldsymbol{\beta}, 1_{\mathbb{L}}\right]=\left\{\left(\alpha_{1}, \alpha_{2}\right) \in \mathbb{L}: \alpha_{1} \geq \beta_{1} \& \alpha_{2} \leq \beta_{2}\right\} .}
\end{aligned}
$$

TheOREM 3.4. Let $f:[0,1] \rightarrow[0,1]$ be a continuous and decreasing function satisfying $f(1)=0$, let $\varphi:[0, f(0)] \rightarrow[0,1]$ be an order isomorphism, and let $Y=\left\{y_{n}\right\}_{n \in \mathbb{N}} \subset[0,1]$ be a decreasing sequence such that $y_{1}=f(0)$ and $\operatorname{Inf}_{n \in \mathbb{N}}\left\{y_{n}\right\}=0$. We consider the function $\mathcal{C}_{\varphi, f, Y}: \mathbb{L}^{X} \rightarrow[0,1]$ defined for each $\chi=(\mu, \nu) \in \mathbb{L}^{X}$ by

$$
\mathcal{C}_{\varphi, f, Y}(\chi)= \begin{cases}0 & \text { if } \chi \in \mathbb{L}_{0}^{X} \\ \varphi\left(y_{n}\right) & \text { if } \operatorname{Sup}_{x \in X} \chi(x) \in R_{n},\end{cases}
$$

where $R_{1}=\left[0_{\mathbb{\imath}},\left(0, y_{1}\right)\right]$ and $R_{n}=\left[0_{\mathbb{L}},\left(f^{-1}\left(y_{n}\right), y_{n}\right)\right] \backslash\left[0_{\mathbb{L}},\left(f^{-1}\left(y_{n-1}\right), y_{n-1}\right)\right]$ for all $n>1$ (see Figure 2(a)). Then $\mathcal{C}_{\varphi, f, Y} \in \mathcal{C} \mathcal{M}_{c s c}\left(\mathbb{L}^{X}\right)$.

THEOREM 3.5. Let $f:[0,1] \rightarrow[0,1]$ be a continuous and decreasing function satisfying $f(1)=0$, let $\varphi:[0, f(0)] \rightarrow[0,1]$ be an order isomorphism, and let $Y=\left\{y_{n}\right\}_{n \in \mathbb{N}} \subset[0,1]$ be a decreasing sequence such that $y_{1}=f(0)$ and $\operatorname{Inf}_{n \in \mathbb{N}}\left\{y_{n}\right\}=0$. We consider the function $\mathcal{C}^{\varphi, f, Y}: \mathbb{L}^{X} \rightarrow[0,1]$ defined for each $\chi=(\mu, \nu) \in \mathbb{L}^{X}$ by

$$
\mathcal{C}^{\varphi, f, Y}(\chi)= \begin{cases}0 & \text { if } \chi \in \mathbb{L}_{0}^{X} \\ \varphi\left(y_{n}\right) & \text { if } \operatorname{Inf}_{x \in X} \chi(x) \in R_{n},\end{cases}
$$

where $R_{1}=\mathbb{L} \backslash\left[\left(0, y_{1}\right), 1_{\mathbb{L}}\right]$ and for all $n>1$,

$$
\begin{aligned}
R_{n}= & {\left[\left(f^{-1}\left(y_{n-1}\right), y_{n-1}\right), 1_{\mathbb{L}}\right] } \\
& \backslash\left(\left[\left(f^{-1}\left(y_{n}\right), y_{n}\right), 1_{\mathbb{}}\right] \cup\left\{\left(\alpha_{1}, 0\right) \in \mathbb{L}: f^{-1}\left(y_{n-1}\right) \leq \alpha_{1} \leq f^{-1}\left(y_{n}\right)\right\}\right)
\end{aligned}
$$

(see Figure 2(b)). Then $\mathcal{C}^{\varphi, f, Y} \in \mathcal{C} \mathcal{M}^{c s c}\left(\mathbb{L}^{X}\right)$.
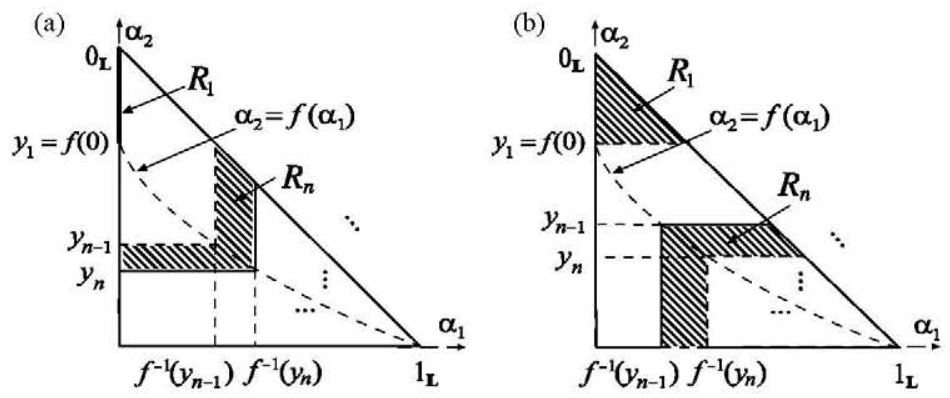

Figure 2. Construction of the regions $R_{n}$ in Theorems 3.4 and 3.5 . 
Under the hypotheses in the above theorems, $\mathcal{C}_{\varphi, f, Y}$ and $\mathcal{C}^{\varphi, f, Y}$ satisfy $\mathcal{C}_{\varphi, f, Y} \in$ $\mathcal{C} \mathcal{M}_{c s c}\left(\mathbb{L}^{X}\right) \subset \mathcal{C} \mathcal{M}_{s c}\left(\mathbb{L}^{X}\right)$ and $\mathcal{C}^{\varphi, f, Y} \in \mathcal{C} \mathcal{M}^{c s c}\left(\mathbb{L}^{X}\right) \subset \mathcal{C} \mathcal{M}^{s c}\left(\mathbb{L}^{X}\right)$. Now let us see what kind of continuity they do not satisfy.

PROPOSITION 3.6. Let $f:[0,1] \rightarrow[0,1]$ be a continuous and decreasing function satisfying $f(1)=0$, let $\varphi:[0, f(0)] \rightarrow[0,1]$ be an order isomorphism, and let $Y=\left\{y_{n}\right\}_{n \in \mathbb{N}} \subset[0,1]$ be a decreasing sequence such that $y_{1}=f(0)$ and $\operatorname{Inf}_{n \in \mathbb{N}}\left\{y_{n}\right\}=0$. Then the following is satisfied.

(a) $\mathcal{C}_{\varphi, f, Y} \notin \mathcal{C} \mathcal{M}^{s c}\left(\mathbb{L}^{X}\right)$ and therefore $\mathcal{C}_{\varphi, f, Y} \notin \mathcal{C} \mathcal{M}^{c s c}\left(\mathbb{L}^{X}\right)$.

(b) $\mathcal{C}^{\varphi, f, Y} \notin \mathcal{C M}_{s c}\left(\mathbb{L}^{X}\right)$ and therefore $\mathcal{C}^{\varphi, f, Y} \notin \mathcal{C} \mathcal{M}_{c s c}\left(\mathbb{L}^{X}\right)$.

Proof. Again, we are going to prove only (a) because the proof of (b) is similar. As $y_{1}=f(0)>0$, we consider $n_{0} \in \mathbb{N}$ such that $1 / n_{0} \leq y_{1}$ and let $\left\{\chi^{n}\right\}_{n \geq n_{0}}$ be the chain (and then it is a lower semilattice too) defined for all $x \in X$ by $\chi^{n}(x)=\left(0, y_{1}-1 / n\right)$; then $\left(\operatorname{Inf}_{n \geq n_{0}} \chi^{n}\right)(x)=\left(0, y_{1}\right)$ for all $x \in X$. Thus, $\mathcal{C}_{\varphi, f, Y}\left(\operatorname{Inf}_{n \geq n_{0}} \chi^{n}\right)=\varphi\left(y_{1}\right)=1$. Nevertheless, as there is $N \in \mathbb{N}$ such that $y_{1}>y_{1}-1 / n \geq y_{2}$ for all $n \geq N$, then $\mathcal{C}_{\varphi, f, Y}\left(\chi^{n}\right)=\varphi\left(y_{2}\right)$ for all $n \geq N$, and $\mathcal{C}_{\varphi, f, Y}\left(\chi^{n}\right)<\varphi\left(y_{2}\right)$ for all $n_{0} \leq n<N$. Therefore, $\operatorname{Sup}_{n \geq n_{0}} \mathcal{C}_{\varphi, f, Y}\left(\chi^{n}\right)=$ $\varphi\left(y_{2}\right)<\varphi\left(y_{1}\right)$.

The last pair of families of contradiction measures that we look at in this section are two families of functions taking values in the whole interval $[0,1]$. One of them comprises measures that are completely semicontinuous from below and the other one contains measures that are completely semicontinuous from above.

THEOREM 3.7. Let $f:[0,1] \rightarrow[0,1]$ be a continuous and decreasing function satisfying $f(1)=0$ and let $\varphi:[0, f(0)] \rightarrow[0,1]$ be an order isomorphism. Then the function $\mathcal{C}_{\varphi, f}:$ $\mathbb{L}^{X} \rightarrow[0,1]$ defined for each $\chi=(\mu, \nu) \in \mathbb{L}^{X}$ by

$$
\mathcal{C}_{\varphi, f}(\chi)=\operatorname{Inf}_{x \in X} \varphi(\operatorname{Min}\{(f \circ \mu)(x), \nu(x)\})
$$

satisfies $\mathcal{C}_{\varphi, f} \in \mathcal{C} \mathcal{M}_{c s c}\left(\mathbb{L}^{X}\right)$.

Proof. Let us confirm the axioms:

(c.i) $\mathcal{C}_{\varphi, f}\left(\chi^{0_{\llcorner}}\right)=\varphi(\operatorname{Min}\{f(0), 1\})=1$;

(c.ii) let $\chi=(\mu, \nu) \in \mathbb{L}^{X}$ be an $\mathbb{L}$-normal set, as $\varphi$ is continuous then

$\mathcal{C}_{\varphi, f}(\chi)=\varphi\left(\operatorname{Min}\left\{\operatorname{Inf}_{x \in X}(f \circ \mu)(x), \operatorname{Inf}_{x \in X} \nu(x)\right\}\right)=\varphi\left(\operatorname{Min}\left\{\operatorname{Inf}_{x \in X}(f \circ \mu)(x), 0\right\}\right)=0 ;$

(c.iv) let $\left\{\chi^{i}\right\}_{i \in \mathcal{I}} \subset \mathbb{L}^{X}$, where $\chi^{i}=\left(\mu_{i}, \nu_{i}\right)$ for all $i \in \mathcal{I}$, because of the properties of the functions $\varphi$ and $f$, we have

$$
\begin{aligned}
\mathcal{C}_{\varphi, f}\left(\operatorname{Sup}_{i \in \mathcal{I}} \chi^{i}\right) & =\operatorname{Inf}_{x \in X} \varphi\left(\operatorname{Min}\left\{\left(f \circ \operatorname{Sup}_{i \in \mathcal{I}} \mu_{i}\right)(x), \operatorname{Inf}_{i \in \mathcal{I}} \nu_{i}(x)\right\}\right) \\
& =\operatorname{Inf}_{x \in X} \varphi\left(\operatorname{Min}\left\{\operatorname{Inf}_{i \in \mathcal{I}}\left(f \circ \mu_{i}\right)(x), \operatorname{Inf}_{i \in \mathcal{I}} \nu_{i}(x)\right\}\right) \\
& =\operatorname{Inf}_{i \in \mathcal{I}} \operatorname{Inf}_{x \in X} \varphi\left(\operatorname{Min}\left\{\left(f \circ \mu_{i}\right)(x), \nu_{i}(x)\right\}\right)=\operatorname{Inf}_{i \in \mathcal{I}} \mathcal{C}_{\varphi, f}\left(\chi^{i}\right) .
\end{aligned}
$$



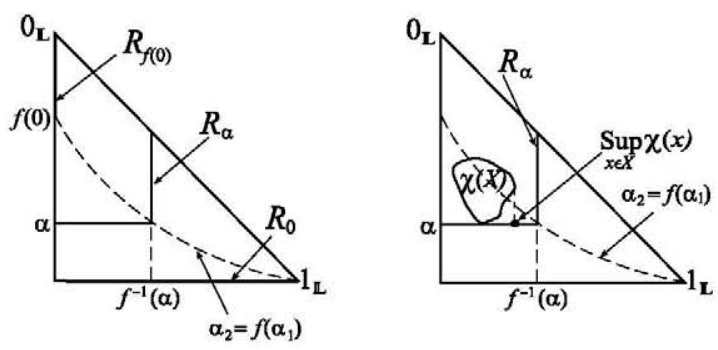

Figure 3. Geometrical interpretation of the measures constructed in Theorem 3.7.

\section{A geometrical interpretation}

We can write

$$
\mathcal{C}_{\varphi, f}(\chi)=\varphi\left(\operatorname{Min}\left\{f\left(\operatorname{Sup}_{x \in X} \mu(x)\right), \operatorname{Inf}_{x \in X} \nu(x)\right\}\right),
$$

so if we denote $\alpha=\operatorname{Min}\left\{f\left(\operatorname{Sup}_{x \in X} \mu(x)\right), \operatorname{Inf}_{x \in X} \nu(x)\right\}$, then either $\operatorname{Sup}_{x \in X} \mu(x)=f^{-1}(\alpha)$ and $\operatorname{Inf}_{x \in X} \nu(x) \geq \alpha$ or $\operatorname{Sup}_{x \in X} \mu(x) \leq f^{-1}(\alpha)$ and $\operatorname{Inf}_{x \in X} \nu(x)=\alpha$. Thus, we consider the square region for which the vertex is located on the curve $\alpha_{2}=f\left(\alpha_{1}\right)$ (see Figure 3) as follows:

$$
R_{\alpha}=\left\{\left(f^{-1}(\alpha), \alpha_{2}\right) \in \mathbb{L}: \alpha_{2} \geq \alpha\right\} \cup\left\{\left(\alpha_{1}, \alpha\right) \in \mathbb{L}: \alpha_{1} \leq f^{-1}(\alpha)\right\},
$$

for all $\alpha \in[0, f(0)]$. Hence, the following statement is satisfied.

$$
\mathcal{C}_{\varphi, f}(\chi)=\varphi(\alpha) \Longleftrightarrow \operatorname{Sup}_{x \in X} \chi(x) \in R_{\alpha} .
$$

Similarly, we can obtain the following result.

THEOREM 3.8. Let $f:[0,1] \rightarrow[0,1]$ be a continuous and decreasing function satisfying $f(1)=0$ and let $\varphi:[0, f(0)] \rightarrow[0,1]$ be an order isomorphism. Let $\mathcal{C}^{\varphi, f}: \mathbb{L}^{X} \rightarrow[0,1]$ be the function defined for each $\chi=(\mu, \nu) \in \mathbb{L}^{X}$ by

$$
\mathcal{C}^{\varphi, f}(\chi)= \begin{cases}0 & \text { if } \chi \in \mathbb{L}_{0}^{X} \\ 1 & \text { if } \chi \notin \mathbb{L}_{0}^{X} \text { and } \operatorname{Sup}_{x \in X} \nu(x) \geq f(0) \\ \operatorname{Sup}_{x \in X} \varphi(\operatorname{Max}\{(f \circ \mu)(x), \nu(x)\}) & \text { otherwise. }\end{cases}
$$

Then $\mathcal{C}^{\varphi, f} \in \mathcal{C} \mathcal{M}^{c s c}\left(\mathbb{L}^{X}\right)$.

\section{A geometrical interpretation}

Let us suppose that $\chi \in \mathbb{L}$ is not $\mathbb{L}$-normal and $\operatorname{Sup}_{x \in X} \nu(x)<f(0)$, thus

$$
\mathcal{C}^{\varphi, f}(\chi)=\varphi\left(\operatorname{Max}\left\{f\left(\operatorname{Inf}_{x \in X} \mu(x)\right), \operatorname{Sup}_{x \in X} \nu(x)\right\}\right)
$$

and if $\alpha=\operatorname{Max}\left\{f\left(\operatorname{Inf}_{x \in X} \mu(x)\right), \operatorname{Sup}_{x \in X} \nu(x)\right\}$ then

$$
\mathcal{C}^{\varphi, f}(\chi)=\varphi(\alpha) \Longleftrightarrow \operatorname{Inf}_{x \in X} \chi(x) \in R_{\alpha},
$$



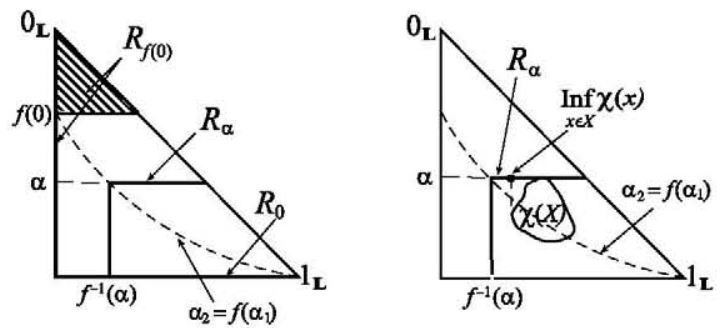

Figure 4. Geometrical interpretation of the measures constructed in Theorem 3.8.

where (see Figure 4)

$$
R_{\alpha}=\left\{\left(f^{-1}(\alpha), \alpha_{2}\right) \in \mathbb{L}: 0<\alpha_{2} \leq \alpha\right\} \cup\left\{\left(\alpha_{1}, \alpha\right) \in \mathbb{L}: \alpha_{1} \geq f^{-1}(\alpha)\right\},
$$

for each $\alpha \in(0, f(0))$. Moreover, $\mathcal{C}^{\varphi, f}(\chi)=\varphi(0)$ if and only if $\operatorname{Inf}_{x \in X} \chi(x) \in R_{0}=$ $[0,1] \times\{0\}$ and $\mathcal{C}^{\varphi, f}(\chi)=\varphi(f(0))$ if and only if $\operatorname{Inf}_{x \in X} \chi(x) \in R_{f(0)}=\left\{\left(\alpha_{1}, \alpha_{2}\right) \in \mathbb{L} \backslash\right.$ $\{(0,0)\}: \alpha_{2} \geq f(0)$ or $\left.\alpha_{1}=0\right\}$.

The geometrical interpretations of Theorems 3.7 and 3.8 show that the measures $\mathcal{C}_{\varphi, f}$ and $\mathcal{C}^{\varphi, f}$ can be interpreted as a limit step of the measures $\mathcal{C}_{\varphi, f, Y}$ and $\mathcal{C}^{\varphi, f, Y}$, respectively (see Figures 2-4).

Example 3.9. To illustrate the above theorems with specific examples, consider again the family $\left\{N_{\lambda}\right\}_{\lambda \in(-1, \infty)}$ of Sugeno's negations and the family $\left\{N_{\gamma}\right\}_{\gamma \in(0, \infty)}$ of Yager's negations. Thus, if $\varphi_{\gamma}(\alpha)=\alpha^{\gamma}$ and $f=N_{\gamma}$, with $\gamma \in(0, \infty)$, or $f=N_{\lambda}$ we have the following.

(a) The measures $\mathcal{C}_{\varphi_{\gamma}, N_{\lambda}}, \mathcal{C}_{\varphi_{\gamma}, N_{\gamma}}: \mathbb{L}^{X} \rightarrow[0,1]$ defined for each $\chi=(\mu, \nu) \in \mathbb{L}^{X}$ by

$$
\begin{aligned}
& \mathcal{C}_{\varphi_{\gamma}, N_{\lambda}}(\chi)=\operatorname{Inf}_{x \in X}\left(\operatorname{Min}\left(\frac{1-\mu(x)}{1+\lambda \mu(x)}, \nu(x)\right)\right)^{\gamma}, \\
& \mathcal{C}_{\varphi_{\gamma}, N_{\gamma}}(\chi)=\operatorname{Inf}_{x \in X} \operatorname{Min}\left(1-\mu(x)^{\gamma}, \nu(x)^{\gamma}\right)
\end{aligned}
$$

are completely semicontinuous from below.

(b) The measures $\mathcal{C}^{\varphi_{\gamma}, N_{\lambda}}, \mathcal{C}^{\varphi_{\gamma}, N_{\gamma}}: \mathbb{L}^{X} \rightarrow[0,1]$ defined for each $\chi=(\mu, \nu) \in \mathbb{L}^{X}$ by

$$
\begin{gathered}
\mathcal{C}^{\varphi_{\gamma}, N_{\lambda}}(\chi)= \begin{cases}0 & \text { if } \chi \in \mathbb{L}_{0}^{X} \\
\operatorname{Sup}_{x \in X}(\operatorname{Max}((1-\mu(x)) /(1+\lambda \mu(x)), \nu(x)))^{\gamma} & \text { if } \chi \in \mathbb{L}^{X} \backslash \mathbb{L}_{0}^{X},\end{cases} \\
\mathcal{C}^{\varphi_{\gamma}, N_{\gamma}}(\chi)= \begin{cases}0 & \text { if } \chi \in \mathbb{L}_{0}^{X} \\
\operatorname{Sup}_{x \in X} \operatorname{Max}\left(1-\mu(x)^{\gamma}, \nu(x)^{\gamma}\right) & \text { if } \chi \in \mathbb{L}^{X} \backslash \mathbb{L}_{0}^{X}\end{cases}
\end{gathered}
$$

are completely semicontinuous from above.

We know that $\mathcal{C}_{\varphi, f} \in \mathcal{C} \mathcal{M}_{c s c}\left(\mathbb{L}^{X}\right) \subset \mathcal{C} \mathcal{M}_{s c}\left(\mathbb{L}^{X}\right)$ and $\mathcal{C}^{\varphi, f} \in \mathcal{C} \mathcal{M}^{c s c}\left(\mathbb{L}^{X}\right) \subset \mathcal{C} \mathcal{M}^{s c}\left(\mathbb{L}^{X}\right)$. Now let us see what kind of continuity $\mathcal{C}_{\varphi, f}$ and $\mathcal{C}^{\varphi, f}$ do not satisfy. 
Proposition 3.10. Let $f:[0,1] \rightarrow[0,1]$ be a continuous and decreasing function satisfying $f(1)=0$ and let $\varphi:[0, f(0)] \rightarrow[0,1]$ be an order isomorphism. Then, the following is satisfied.

(a) If $X$ is an infinite set, $\mathcal{C}_{\varphi, f} \notin \mathcal{C} \mathcal{M}^{s c}\left(\mathbb{L}^{X}\right)$, whence $\mathcal{C}_{\varphi, f} \notin \mathcal{C} \mathcal{M}^{c s c}\left(\mathbb{L}^{X}\right)$.

(b) $\mathcal{C}^{\varphi, f} \notin \mathcal{C} \mathcal{M}_{s c}\left(\mathbb{L}^{X}\right)$, whence $\mathcal{C}^{\varphi, f} \notin \mathcal{C} \mathcal{M}_{c s c}\left(\mathbb{L}^{X}\right)$.

Proof. (a) The idea is the same as in Proposition 3.3. Again, we consider the set of all finite parts of $X, \mathcal{P}_{F}(X)$, and let $\left\{\chi^{A}\right\}_{A \in \mathcal{P}_{F}(X)} \subset \mathbb{L}^{X}$ be the family such that for each $A \in \mathcal{P}_{F}(X)$,

$$
\chi^{A}(x)= \begin{cases}0_{\mathbb{L}} & \text { if } x \in A \\ \left(0, \frac{f(0)}{2}\right) & \text { if } x \notin A .\end{cases}
$$

$\left\{\chi^{A}\right\}_{A \in \mathcal{P}_{F}(X)}$ is a lower semilattice because if $\chi^{A_{1}}, \chi^{A_{2}} \in\left\{\chi^{A}\right\}_{A \in \mathcal{P}_{F}(X)}$, then $\operatorname{Inf}\left\{\chi^{A_{1}}, \chi^{A_{2}}\right\}=\chi^{A_{1} \cup A_{2}} \in\left\{\chi^{A}\right\}_{A \in \mathcal{P}_{F}(X)}$. Thus, $\mathcal{C}_{\varphi, f}\left(\chi^{A}\right)=\varphi(f(0) / 2)<1$ for all $\chi^{A} \in$ $\left\{\chi^{A}\right\}_{A \in \mathcal{P}_{F}(X)}$ and so $\operatorname{Sup}_{A \in \mathcal{P}_{F}(X)} \mathcal{C}_{\varphi, f}\left(\chi^{A}\right)=\varphi(f(0) / 2)<1$. However, $\mathcal{C}_{\varphi, f}\left(\operatorname{Inf}_{A \in \mathcal{P}_{F}(X)} \chi^{A}\right)=$ $\mathcal{C}_{\varphi, f}\left(\chi^{0_{\mathrm{L}}}\right)=1$.

(b) Let $\left\{\chi^{n}\right\}_{n \in \mathbb{N}}$ be the chain (and then it is a upper semilattice too) defined for all $x \in X$ by $\chi^{n}(x)=(0, f(0) / n)$, then $\left(\operatorname{Sup}_{n \in \mathbb{N}} \chi^{n}\right)(x)=(0,0)$ for all $x \in X$. Thus $\mathcal{C}^{\varphi, f}\left(\operatorname{Sup}_{n \in \mathbb{N}} \chi^{n}\right)=0$, as $\operatorname{Sup}_{n \in \mathbb{N}} \chi^{n}$ is $\mathbb{L}$-normal. Nevertheless, $\mathcal{C}^{\varphi, f}\left(\chi^{n}\right)=1$ for all $n \in \mathbb{N}$, and therefore $\operatorname{Inf}_{n \in \mathbb{N}} \mathcal{C}^{\varphi, f}\left(\chi^{n}\right)=1$.

\section{Families of semicontinuous contradiction measures}

In this section, we introduce several families of semicontinuous contradiction measures. Some are functions taking discrete values in $[0,1]$ and the rest take values in the whole interval $[0,1]$. Each family that is semicontinuous from below has a 'dual' family that is semicontinuous from above.

THeOREM 4.1. Let $\varphi:[0,1] \rightarrow[0,1]$ be an order automorphism, let $Y=\left\{y_{n}\right\}_{n \in \mathbb{N}} \subset[0,1]$ be a decreasing sequence such that $\operatorname{Inf}_{n \in \mathbb{N}}\left\{y_{n}\right\}=0$ and $y_{1}=1$, and let $p>0$. We consider the family of regions in $\mathbb{L}$ bordered by the straight lines joining the two points $(-p, 0)$ and $\left(0, y_{n}\right)$, for each $n \in \mathbb{N}$, as follows.

$$
\begin{aligned}
& R_{1}=\left\{0_{\mathbb{L}}\right\} \\
& R_{n}=\left\{\left(\alpha_{1}, \alpha_{2}\right) \in \mathbb{L}: y_{n}+\alpha_{1} \frac{y_{n}}{p} \leq \alpha_{2}<y_{n-1}+\alpha_{1} \frac{y_{n-1}}{p}\right\} \quad \forall n>1 .
\end{aligned}
$$

Then, the following two functions are both contradiction measures that are semicontinuous from below.

(a) For each $\chi \in \mathbb{L}^{X}$,

$$
\mathcal{C}_{\varphi, p, Y}(\chi)= \begin{cases}0 & \text { if } \chi \in \mathbb{L}_{0}^{X} \\ \varphi\left(y_{n}\right) & \text { if } \operatorname{Sup}_{x \in X} \chi(x) \in R_{n}\end{cases}
$$

(b) For each $\chi \in \mathbb{L}^{X}$,

$$
\tilde{\mathcal{C}}_{\varphi, p, Y}(\chi)= \begin{cases}0 & \text { if } \chi \in \mathbb{\mathbb { L }}_{0}^{X} \\ \varphi\left(y_{n}\right) & \text { if } n=\operatorname{Sup}\left\{k \in \mathbb{N}: \chi(X) \cap R_{k} \neq \emptyset\right\} .\end{cases}
$$


On the one hand, the family of (a) is a more general construction than another introduced by Castiñeira and Cubillo (2009). Thus (a) is proven by techniques similar to the proof shown there. Therefore, the proofs are omitted. On the other hand, although the construction in (b) is a new family, the proof of that statement is similar to the proof of (a).

Figure 5 shows what the measures $\mathcal{C}_{\varphi, p, Y}$ and $\tilde{\mathcal{C}}_{\varphi, p, Y}$ are like, emphasizing the difference between them.

As in the above theorem, we obtain the following results.

TheOREM 4.2. Let $\varphi:[0,1] \rightarrow[0,1]$ be an order of automorphism, let $Y=\left\{y_{n}\right\}_{n \in \mathbb{N}} \subset$ $[0,1]$ be a decreasing sequence such that $\operatorname{Inf}_{n \in \mathbb{N}}\left\{y_{n}\right\}=0$ and $y_{1}=1$, and let $p>0$. For each $n \in \mathbb{N}$, we consider

$$
R_{n}=\left\{\left(\alpha_{1}, \alpha_{2}\right) \in \mathbb{L}: y_{n+1}+\alpha_{1} \frac{y_{n+1}}{p}<\alpha_{2} \leq y_{n}+\alpha_{1} \frac{y_{n}}{p}\right\} .
$$

Then, the following two functions are both contradiction measures that are semicontinuous from above.

(a) For each $\chi \in \mathbb{L}^{X}$,

$$
\mathcal{C}^{\varphi, p, Y}(\chi)= \begin{cases}0 & \text { if } \chi \in \mathbb{L}_{0}^{X} \\ \varphi\left(y_{n}\right) & \text { if } \operatorname{Inf}_{x \in X} \chi(x) \in R_{n}\end{cases}
$$

(b) For each $\chi \in \mathbb{L}^{X}$,

$$
\tilde{\mathcal{C}}^{\varphi, p, Y}(\chi)= \begin{cases}0 & \text { if } \chi \in \mathbb{L}_{0}^{X} \\ \varphi\left(y_{n}\right) & \text { if } n=\operatorname{Inf}\left\{k \in \mathbb{N}: \chi(X) \cap R_{k} \neq \emptyset\right\} .\end{cases}
$$

Figure 6 shows a geometrical interpretation of the measures $\mathcal{C}^{\varphi, p, Y}$ and $\tilde{\mathcal{C}}^{\varphi, p, Y}$, emphasizing the difference between them.

Let us present a sufficient condition on an A-IFS for the measures in Theorem 4.1 to be equal, and another one for measures in Theorem 4.2 to be equal.

Proposition 4.3. Let $\varphi, p$, and $Y$ be the same as in the previous theorems, and $\chi=(\mu, \nu) \in \mathbb{R}^{X}$, then the following holds.

(a) If $\{\chi(x)\}_{x \in X}$ is an upper semilattice, then $\mathcal{C}_{\varphi, p, Y}(\chi)=\tilde{\mathcal{C}}_{\varphi, p, Y}(\chi)$ holds.

(b) If $\{\chi(x)\}_{x \in X}$ is a lower semilattice, then $\mathcal{C}^{\varphi, p, Y}(\chi)=\tilde{\mathcal{C}}^{\varphi, p, Y}(\chi)$ holds.

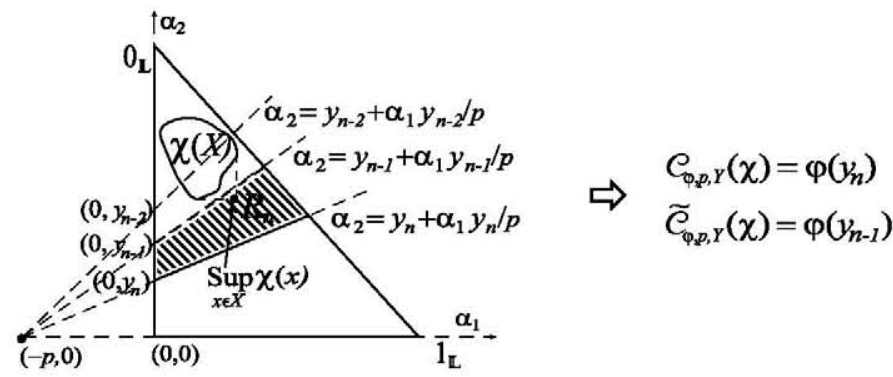

Figure 5. Geometrical interpretation of the measures $\mathcal{C}_{\varphi, p, Y}$ and $\tilde{\mathcal{C}}_{\varphi, p, Y}$. 


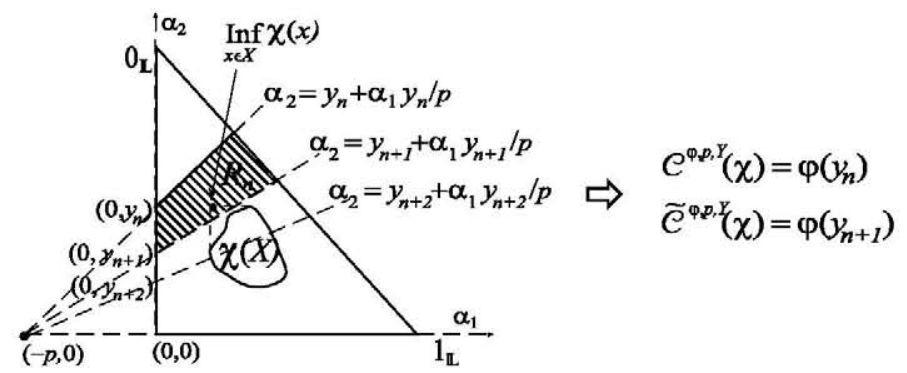

Figure 6. Geometrical interpretation of the measures $\mathcal{C}^{\varphi, p, Y}$ and $\tilde{\mathcal{C}}^{\varphi, p, Y}$.

Proof. Let us only prove part (a) because the proof of (b) is similar to that of (a). First, let us prove that if $\{\chi(x)\}_{x \in X}$ is an upper semilattice, then $\operatorname{Sup}_{x \in X} \chi(x) \in \overline{\chi(X)}$, where $\overline{\chi(X)}$ is the closure of $\chi(X)$ under the usual topology on $\mathbb{R}^{2}$ restricted to $\mathbb{L}$. As $\operatorname{Sup}_{x \in X} \chi(x)=$ $\left(\operatorname{Sup}_{x \in X} \mu(x), \operatorname{Inf}_{x \in X} \nu(x)\right)$, for all $\varepsilon>0$, there exist $x_{1}, x_{2} \in X$ such that

$$
\begin{aligned}
& \mu\left(x_{1}\right)>\operatorname{Sup}_{x \in X} \mu(x)-\frac{\varepsilon}{\sqrt{2}}, \\
& \nu\left(x_{2}\right)<\operatorname{Inf}_{x \in X} \nu(x)+\frac{\varepsilon}{\sqrt{2}} .
\end{aligned}
$$

Besides, as $\{\chi(x)\}_{x \in X}$ is an upper semilattice, there exists $x_{\varepsilon} \in X$ such that

$$
\chi\left(x_{\varepsilon}\right)=\operatorname{Sup}\left\{\chi\left(x_{1}\right), \chi\left(x_{2}\right)\right\} .
$$

Therefore, for all $\varepsilon>0$, there exists $x_{\varepsilon} \in X$ such that $d\left(\operatorname{Sup}_{x \in X} \chi(x), \chi\left(x_{\varepsilon}\right)\right)<\varepsilon, d$ being the Euclidean distance in $\mathbb{R}^{2}$. Thus, $\operatorname{Sup}_{x \in X} \chi(x) \in \overline{\chi(X)}$.

Now, if $\chi$ is $\mathbb{L}$-normal, then $\mathcal{C}_{\varphi, p, Y}(\chi)=\tilde{\mathcal{C}}_{\varphi, p, Y}(\chi)$ is trivially satisfied. Suppose that $\chi$ is not $\mathbb{L}$-normal and $\mathcal{C}_{\varphi, p, Y}(\chi)=\varphi\left(y_{n_{0}}\right)$, for some $n_{0} \in \mathbb{N}$. Thus, $\operatorname{Sup}_{x \in X} \chi(x) \in R_{n_{0}}$, for all $y \in X$ and for all $n \geq n_{0}$, the following holds.

$$
\nu(y) \geq \operatorname{Inf}_{x \in X} \nu(x) \geq y_{n_{0}}+\operatorname{Sup}_{x \in X} \mu(x) \frac{y_{n_{0}}}{p} \geq y_{n_{0}}+\mu(y) \frac{y_{n_{0}}}{p} \geq y_{n}+\mu(y) \frac{y_{n}}{p} .
$$

Hence, $\chi(y) \notin R_{n+1}$ for all $y \in X$ and for all $n \geq n_{0}$, i.e. $\chi(X) \subset \cup_{n=1}^{n_{0}} R_{n}$, thus $\tilde{\mathcal{C}}_{\varphi, p, Y}(\chi) \geq \varphi\left(y_{n_{0}}\right)$.

Moreover, as $R_{n} \cap R_{m}=\emptyset$ for all $n, m \in \mathbb{N}$, then $d\left(\operatorname{Sup}_{x \in X} \chi(x), \cup_{n=1}^{n_{0}-1} R_{n}\right)=d_{0}>0$. For $d_{0}$, there exists $x_{0} \in X$ such that $d\left(\chi\left(x_{0}\right), \operatorname{Sup}_{x \in X} \chi(x)\right)<d_{0}$ since $\operatorname{Sup}_{x \in X} \chi(x) \in$ $\overline{\chi(X)}$. Thus,

$$
\begin{aligned}
d_{0} & =d\left(\operatorname{Sup}_{x \in X} \chi(x), \cup_{n=1}^{n_{0}-1} R_{n}\right) \leq d\left(\operatorname{Sup}_{x \in X} \chi(x), \chi\left(x_{0}\right)\right)+d\left(\chi\left(x_{0}\right), \cup_{n=1}^{n_{0}-1} R_{n}\right) \\
& <d_{0}+d\left(\chi\left(x_{0}\right), \cup_{n=1}^{n_{0}-1} R_{n}\right) .
\end{aligned}
$$

Therefore, $d\left(\chi\left(x_{0}\right), \cup_{n=1}^{n_{0}-1} R_{n}\right)>0$ and so $\chi\left(x_{0}\right) \in R_{n_{0}}$, hence $\tilde{\mathcal{C}}_{\varphi, p, Y}(\chi) \leq \varphi\left(y_{n_{0}}\right)$.

Now, let us see what properties the measures constructed in Theorems 4.1 and 4.2 do not satisfy. 
PRoposition 4.4. Let $\varphi:[0,1] \rightarrow[0,1]$ be an order of automorphism, let $Y=\left\{y_{n}\right\}_{n \in \mathbb{N}} \subset$ $[0,1]$ be a decreasing sequence such that $\operatorname{Inf}_{n \in \mathbb{N}}\left\{y_{n}\right\}=0$ and $y_{1}=1$, and let $p>0$. Then, the following is satisfied.

(a) $\mathcal{C}_{\varphi, p, Y}, \tilde{\mathcal{C}}_{\varphi, p, Y} \notin \mathcal{C} \mathcal{M}_{c s c}\left(\mathbb{L}^{X}\right)$ and $\mathcal{C}^{\varphi, p, Y}, \tilde{\mathcal{C}}^{\varphi, p, Y} \notin \mathcal{C} \mathcal{M}^{c s c}\left(\mathbb{L}^{X}\right)$.

(b) $\mathcal{C}_{\varphi, p, Y}, \tilde{\mathcal{C}}_{\varphi, p, Y} \notin \mathcal{C} \mathcal{M}^{s c}\left(\mathbb{L}^{X}\right)$ and $\mathcal{C}^{\varphi, p, Y}, \tilde{\mathcal{C}}^{\varphi, p, Y} \notin \mathcal{C} \mathcal{M}_{s c}\left(\mathbb{L}^{X}\right)$.

Proof. Let us prove the statements for $\mathcal{C}_{\varphi, p, Y}$ and $\tilde{\mathcal{C}}_{\varphi, p, Y}$; in the same way, we can obtain the results for $\mathcal{C}^{\varphi, p, Y}$ and $\tilde{\mathcal{C}}^{\varphi, p, Y}$.

(a) To show that $\mathcal{C}_{\varphi, p, Y}$ and $\tilde{\mathcal{C}}_{\varphi, p, Y}$ are not completely semicontinuous from below it is sufficient to consider $\chi^{1}, \chi^{2} \in \mathbb{L}^{X}$ such that $\chi^{1}(x)=\left(0, y_{2}\right)$ and $\chi^{2}(x)=\left(\left(p\left(1-y_{2}\right)\right) /(p+\right.$ $\left.\left.y_{2}\right),\left(y_{2}(p+1)\right) /\left(p+y_{2}\right)\right)$ for all $x \in X$. Thus, $\chi^{1}(X), \chi^{2}(X) \subset\left\{\left(\alpha_{1}, \alpha_{2}\right): \alpha_{2}=y_{2}+\right.$ $\left.\alpha_{1}\left(y_{2} / p\right)\right\} \subset R_{2}$ and, however, for all $x \in X$, $\operatorname{Sup}\left\{\chi^{1}, \chi^{2}\right\}(x)=\left(\left(p\left(1-y_{2}\right)\right) /(p+\right.$ $\left.\left.y_{2}\right), y_{2}\right) \notin R_{2}$ as $y_{2}<\left(y_{2}(p+1)\right) /\left(p+y_{2}\right)$ since $0<y_{2}<1$ (see Figure 7). Thus,

$$
\tilde{\mathcal{C}}_{\varphi, p, Y}\left(\chi^{1}\right)=\mathcal{C}_{\varphi, p, Y}\left(\chi^{1}\right)=\mathcal{C}_{\varphi, p, Y}\left(\chi^{2}\right)=\tilde{\mathcal{C}}_{\varphi, p, Y}\left(\chi^{2}\right)=\varphi\left(y_{2}\right)
$$

and, nevertheless,

$$
\mathcal{C}_{\varphi, p, Y}\left(\operatorname{Sup}\left\{\chi^{1}, \chi^{2}\right\}\right)=\tilde{\mathcal{C}}_{\varphi, p, Y}\left(\operatorname{Sup}\left\{\chi^{1}, \chi^{2}\right\}\right)=\varphi\left(y_{n}\right)
$$

for some $n>2$. Hence, $\mathcal{C}_{\varphi, p, Y} \notin \mathcal{C} \mathcal{M}_{c s c}\left(\mathbb{L}^{X}\right)$ and $\tilde{\mathcal{C}}_{\varphi, p, Y} \notin \mathcal{C} \mathcal{M}_{c s c}\left(\mathbb{L}^{X}\right)$.

(b) To show that $\mathcal{C}_{\varphi, p, Y}$ and $\tilde{\mathcal{C}}_{\varphi, p, Y}$ are not semicontinuous from above (and, therefore, not completely semicontinuous from above), we consider $n_{0} \in \mathbb{N}$ such that $1-y_{n} \geq y_{2}$ for all $n \geq n_{0}$ and the chain $\left\{\chi^{n}\right\}_{n \geq n_{0}}$ defined for all $x \in X$ by $\chi^{n}(x)=\left(0,1-y_{n}\right)$. Thus, $\left(\operatorname{Inf}_{n \geq n_{0}} \chi^{n}\right)(x)=0_{\mathbb{L}}$ for all $x \in X$ and so $\mathcal{C}_{\varphi, p, Y}\left(\operatorname{Inf}_{n \geq n_{0}} \chi^{n}\right)=\tilde{\mathcal{C}}_{\varphi, p, Y}\left(\operatorname{Inf}_{n \geq n_{0}} \chi^{n}\right)=1$. Nevertheless, $\quad \operatorname{Sup}_{n \geq n_{0}} \mathcal{C}_{\varphi, p, Y}\left(\chi^{n}\right)=\operatorname{Sup}_{n \geq n_{0}} \tilde{\mathcal{C}}_{\varphi, p, Y}\left(\chi^{n}\right)=\varphi\left(y_{2}\right)<1$ as $\mathcal{C}_{\varphi, p, Y}\left(\chi^{n}\right)=$ $\tilde{\mathcal{C}}_{\varphi, p, Y}\left(\chi^{n}\right)=\varphi\left(y_{2}\right)$ for all $n \geq n_{0}$. Therefore, $\mathcal{C}_{\varphi, p, Y} \notin \mathcal{C} \mathcal{M}^{s c}\left(\mathbb{L}^{X}\right)$ and $\tilde{\mathcal{C}}_{\varphi, p, Y} \notin$ $\mathcal{C} \mathcal{M}^{s c}\left(\mathbb{L}^{X}\right)$.

Theorem 4.5. Let $\varphi:[0,1] \rightarrow[0,1]$ be an order of automorphism, then for all $p>0$, the function $\mathcal{C}_{\varphi, p}: \mathbb{L}^{X} \rightarrow[0,1]$ defined for each $\chi=(\mu, \nu) \in \mathbb{L}^{X}$ by

$$
\mathcal{C}_{\varphi, p}(\chi)=\varphi\left(\frac{p \operatorname{Inf}_{x \in X} \nu(x)}{p+\operatorname{Sup}_{x \in X} \mu(x)}\right)
$$

is a contradiction measure that is semicontinuous from below.

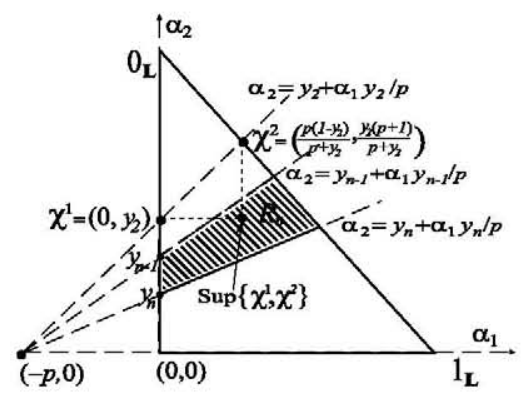

Figure 7. Proof of Proposition 4.4(a). 
Proof. Let us note that $\mathcal{C}_{\varphi, p}$ is well defined as $0 \leq p \operatorname{Inf}_{x \in X} \nu(x) \leq p \leq p+\operatorname{Sup}_{x \in X} \mu(x)$. To prove that $\mathcal{C}_{\varphi, p} \in \mathcal{C} \mathcal{M}_{s c}\left(\mathbb{L}^{X}\right)$, we only need to show that the function $\mathcal{C}_{p}(\chi)=$ $\left(p \operatorname{Inf}_{x \in X} \nu(x)\right) /\left(p+\operatorname{Sup}_{x \in X} \mu(x)\right)$ is a contradiction measure that is semicontinuous from below, since the axioms (c.i), (c.ii), and (c.iv) are preserved under automorphism.

Trivially, $\mathcal{C}_{p}$ satisfies the axioms (c.i)-(c.iii). Let us see the axiom (c.vi). Let $\left\{\chi^{i}\right\}_{i \in \mathcal{I}} \subset \mathbb{Q}^{X}$ be an upper semilattice $\chi^{i}=\left(\mu_{i}, \nu_{i}\right)$ being for each $i \in \mathcal{I}$. Due to the antimonotonicity of $\mathcal{C}_{p}, \operatorname{Inf}_{i \in \mathcal{I}} \mathcal{C}_{p}\left(\chi^{i}\right) \geq \mathcal{C}_{p}\left(\operatorname{Sup}_{i \in \mathcal{I}} \chi^{i}\right)$ holds. To verify the other inequality, we consider $\varepsilon>0$, thus there exist $i_{1}, i_{2} \in \mathcal{I}$ and there exist $x_{1}, x_{2} \in X$ such that

$$
\begin{gathered}
\operatorname{Inf}_{x \in X} \operatorname{Inf}_{i \in \mathcal{I}} \nu_{i}(x) \leq \nu_{i_{1}}\left(x_{1}\right)<\operatorname{Inf}_{x \in X} \operatorname{Inf}_{i \in \mathcal{I}} \nu_{i}(x)+\varepsilon, \\
\operatorname{Sup}_{x \in X} \operatorname{Sup}_{i \in \mathcal{I}} \mu_{i}(x) \geq \mu_{i_{2}}\left(x_{2}\right)>\operatorname{Sup}_{x \in X} \operatorname{Sup}_{i \in \mathcal{I}} \nu_{i}(x)-\varepsilon .
\end{gathered}
$$

As $\left\{\chi^{i}\right\}_{i \in \mathcal{I}}$ is an upper semilattice, there exists $i_{\varepsilon} \in \mathcal{I}$ such that $\chi^{i_{\varepsilon}}=\operatorname{Sup}\left\{\chi^{i_{1}}, \chi^{i_{2}}\right\}$ and thus the following holds.

$$
\begin{gathered}
\operatorname{Inf}_{x \in X} \nu_{i_{\varepsilon}}(x) \leq \nu_{i_{\varepsilon}}\left(x_{1}\right) \leq \nu_{i_{1}}\left(x_{1}\right)<\operatorname{Inf}_{x \in X} \operatorname{Inf}_{i \in \mathcal{I}} \nu_{i}(x)+\varepsilon . \\
\operatorname{Sup}_{x \in X} \mu_{i_{\varepsilon}}(x) \geq \mu_{i_{\varepsilon}}\left(x_{2}\right) \geq \mu_{i_{2}}\left(x_{2}\right)>\operatorname{Sup}_{x \in X} \operatorname{Sup}_{i \in \mathcal{I}} \mu_{i}(x)-\varepsilon .
\end{gathered}
$$

Then,

$$
\mathcal{C}_{p}\left(\chi^{i_{\varepsilon}}\right)=\frac{p \operatorname{Inf}_{x \in X} \nu_{i_{\varepsilon}}(x)}{p+\operatorname{Sup}_{x \in X} \mu_{i_{\varepsilon}}(x)}<\frac{p\left(\operatorname{Inf}_{x \in X} \operatorname{Inf}_{i \in \mathcal{I}} \nu_{i}(x)+\varepsilon\right)}{p+\operatorname{Sup}_{x \in X} \operatorname{Sup}_{i \in \mathcal{I}} \mu_{i}(x)-\varepsilon} .
$$

Taking into account that $\left\{i_{\varepsilon}: \varepsilon\right\} \subset \mathcal{I}$, we arrive at

$$
\begin{aligned}
\operatorname{Inf}_{i \in \mathcal{I}} \mathcal{C}_{p}\left(\chi^{i}\right) & \leq \operatorname{Inf}_{\varepsilon>0} \mathcal{C}_{p}\left(\chi^{i_{c}}\right) \leq \operatorname{Inf}_{\varepsilon>0} \frac{p\left(\operatorname{Inf}_{x \in X} \operatorname{Inf}_{i \in \mathcal{I}} \nu_{i}(x)+\varepsilon\right)}{p+\operatorname{Sup}_{x \in X} \operatorname{Sup}_{i \in \mathcal{I}} \mu_{i}(x)-\varepsilon} \\
& =\frac{p\left(\operatorname{Inf}_{x \in X} \operatorname{Inf}_{i \in \mathcal{I}} \nu_{i}(x)\right)}{p+\operatorname{Sup}_{x \in X} \operatorname{Sup}_{i \in \mathcal{I}} \mu_{i}(x)}=\mathcal{C}_{p}\left(\operatorname{Sup}_{i \in \mathcal{I}} \chi^{i}\right)
\end{aligned}
$$

A geometrical interpretation

$$
\mathcal{C}_{\varphi, p}(\chi)=\varphi\left(\frac{p \operatorname{Inf}_{x \in X} \nu(x)}{p+\operatorname{Sup}_{x \in X} \mu(x)}\right)=\varphi(\alpha)
$$

if and only if $(\alpha / p) \operatorname{Sup}_{x \in X} \mu(x)+\alpha=\operatorname{Inf}_{x \in X} \nu(x)$. This is equivalent to

$$
\operatorname{Sup}_{x \in X} \chi(x)=\left(\operatorname{Sup}_{x \in X} \mu(x), \operatorname{Inf}_{x \in X} \nu(x)\right)
$$

staying on the straight line $\alpha_{2}=\alpha+\alpha_{1} \alpha / p$, that is (see Figure 8),

$$
\mathcal{C}_{\varphi, p}(\chi)=\varphi(\alpha) \Longleftrightarrow \operatorname{Sup}_{x \in X} \chi(x) \in R_{\alpha}=\left\{\left(\alpha_{1}, \alpha_{2}\right) \in \mathbb{L}: \alpha_{2}=\alpha+\alpha_{1} \alpha / p\right\} .
$$




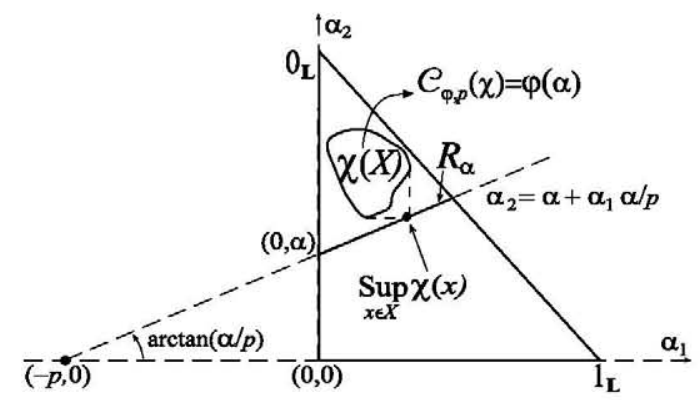

Figure 8. Geometrical interpretation of the measure constructed in Theorem 4.5.

THEOREM 4.6. Let $\varphi:[0,1] \rightarrow[0,1]$ be an order automorphism, then for all $p>0$, the function $\tilde{\mathcal{C}}_{\varphi, p}: \mathbb{L}^{X} \rightarrow[0,1]$ defined for each $\chi=(\mu, \nu) \in \mathbb{L}^{X}$ by

$$
\tilde{\mathcal{C}}_{\varphi, p}(\chi)=\operatorname{Inf}_{x \in X} \varphi\left(\frac{p \nu(x)}{p+\mu(x)}\right)
$$

is a contradiction measure that is semicontinuous from below.

Proof. Again it is sufficient to prove that $\tilde{\mathcal{C}}_{p} \in \mathcal{C} \mathcal{M}_{s c}\left(\mathbb{L}^{X}\right)$, where $\tilde{\mathcal{C}}_{p}(\chi)=\operatorname{Inf}_{x \in X} p \nu(x)$ / $(p+\mu(x))$ for each $\chi=(\mu, \nu) \in \mathbb{L}^{X}$. The axioms (c.i) and (c.ii) are trivially satisfied by $\tilde{\mathcal{C}}_{p}$.

To show (c.vi), we consider any upper semilattice $\left\{\chi^{i}\right\}_{i \in \mathcal{I}} \subset \mathbb{L}^{X}$, where $\chi^{i}=\left(\mu_{i}, \nu_{i}\right)$ for each $i \in \mathcal{I}$. Let us prove that for all $x \in X$ the following is satisfied:

$$
\operatorname{Inf}_{i \in \mathcal{I}} \frac{\nu_{i}(x)}{p+\mu_{i}(x)}=\frac{\operatorname{Inf}_{i \in \mathcal{I}} \nu_{i}(x)}{p+\operatorname{Sup}_{i \in \mathcal{I}} \mu_{i}(x)} .
$$

Let $x \in X$ be a fixed element,

$$
\frac{\operatorname{Inf}_{i \in \mathcal{I}} \nu_{i}(x)}{p+\operatorname{Sup}_{i \in \mathcal{I}} \mu_{i}(x)} \leq \frac{\nu_{j}(x)}{p+\mu_{j}(x)}
$$

holds for all $j \in \mathcal{I}$. Let us see that the previous lower bound of the set $\left\{\nu_{i}(x) /(p+\right.$ $\left.\left.\mu_{i}(x)\right)\right\}_{i \in \mathcal{I}} \subset[0,1]$ is its greatest lower bound. To do this, we must find for each $\varepsilon>0$ an index $i_{\varepsilon} \in \mathcal{I}$ such that

$$
\frac{\operatorname{Inf}_{i \in \mathcal{I}} \nu_{i}(x)}{p+\operatorname{Sup}_{i \in \mathcal{I}} \mu_{i}(x)} \leq \frac{\nu_{i_{\varepsilon}}(x)}{p+\mu_{i_{c}}(x)}<\frac{\operatorname{Inf}_{i \in \mathcal{I}} \nu_{i}(x)}{p+\operatorname{Sup}_{i \in \mathcal{I}} \mu_{i}(x)}+\varepsilon .
$$

From

$$
\frac{\operatorname{Inf}_{i \in \mathcal{I}} \nu_{i}(x)}{p+\operatorname{Sup}_{i \in \mathcal{I}} \mu_{i}(x)}<\frac{\operatorname{Inf}_{i \in \mathcal{I}} \nu_{i}(x)}{p+\operatorname{Sup}_{i \in \mathcal{I}} \mu_{i}(x)}+\varepsilon
$$

we can infer

$$
\frac{\operatorname{Inf}_{i \in \mathcal{I}} \nu_{i}(x)\left(p+\operatorname{Sup}_{i \in \mathcal{I}} \mu_{i}(x)\right)}{\operatorname{Inf}_{i \in \mathcal{I}} \nu_{i}(x)+\varepsilon\left(p+\operatorname{Sup}_{i \in \mathcal{I}} \mu_{i}(x)\right)}-p<\operatorname{Sup}_{i \in \mathcal{I}} \mu_{i}(x)
$$


thus, there exists $j \in \mathcal{I}$ such that

$$
\frac{\operatorname{Inf}_{i \in \mathcal{I}} \nu_{i}(x)\left(p+\operatorname{Sup}_{i \in \mathcal{I}} \mu_{i}(x)\right)}{\operatorname{Inf}_{i \in \mathcal{I}} \nu_{i}(x)+\varepsilon\left(p+\operatorname{Sup}_{i \in \mathcal{I}} \mu_{i}(x)\right)}-p<\mu_{j}(x) \leq \operatorname{Sup}_{i \in \mathcal{I}} \mu_{i}(x) .
$$

It follows from the inequality on the left in (2) that

$$
\operatorname{Inf}_{i \in \mathcal{I}} \nu_{i}(x)<\left(p+\mu_{j}(x)\right)\left(\frac{\operatorname{Inf}_{i \in \mathcal{I}} \nu_{i}(x)}{p+\operatorname{Sup}_{i \in \mathcal{I}} \mu_{i}(x)}+\varepsilon\right),
$$

thus, there exists $k \in \mathcal{I}$ such that

$$
\operatorname{Inf}_{i \in \mathcal{I}} \nu_{i}(x) \leq \nu_{k}(x)<\left(p+\mu_{j}(x)\right)\left(\frac{\operatorname{Inf}_{i \in \mathcal{I}} \nu_{i}(x)}{p+\operatorname{Sup}_{i \in \mathcal{I}} \mu_{i}(x)}+\varepsilon\right) .
$$

Now, as $\left\{\chi^{i}\right\}_{i \in \mathcal{I}}$ is an upper semilattice, there exists $i_{\varepsilon} \in \mathcal{I}$ such that $\chi^{i_{c}}=\operatorname{Sup}\left\{\chi^{j}, \chi^{k}\right\}$. From (3) and taking into account that $\mu_{j}(x) \leq \mu_{i_{\varepsilon}}(x)$ and $\nu_{k}(x) \geq \nu_{i_{\varepsilon}}(x)$, we obtain

$$
\frac{\nu_{i_{c}}(x)}{p+\mu_{i_{c}}(x)} \leq \frac{\nu_{k}(x)}{p+\mu_{j}(x)}<\frac{\operatorname{Inf}_{i \in \mathcal{I}} \nu_{i}(x)}{p+\operatorname{Sup}_{i \in \mathcal{I}} \mu_{i}(x)}+\varepsilon .
$$

Hence, equality (1) is proved and therefore

$$
\begin{aligned}
\operatorname{Inf}_{i \in \mathcal{I}} \tilde{\mathcal{C}}_{p}\left(\chi^{i}\right) & =\operatorname{Inf}_{i \in \mathcal{I}} \operatorname{Inf}_{x \in X} \frac{p \nu_{i}(x)}{p+\mu_{i}(x)}=\operatorname{Inf}_{x \in X} p \operatorname{Inf}_{i \in \mathcal{I}} \frac{\nu_{i}(x)}{p+\mu_{i}(x)} \\
& =\operatorname{Inf}_{x \in X} \frac{p \operatorname{Inf}_{i \in \mathcal{I}} \nu_{i}(x)}{p+\operatorname{Sup}_{i \in \mathcal{I}} \mu_{i}(x)}=\tilde{\mathcal{C}}_{p}\left(\operatorname{Sup}_{i \in \mathcal{I}} \chi^{i}\right) .
\end{aligned}
$$

\section{A geometrical interpretation}

Again, if $R_{\gamma}=\left\{\left(\alpha_{1}, \alpha_{2}\right) \in \mathbb{L}: \alpha_{2}=\gamma+\alpha_{1} \gamma / p\right\}$, then

$$
\tilde{\mathcal{C}}_{\varphi, p}(\chi)=\varphi(\alpha) \Longleftrightarrow \alpha=\operatorname{Inf}\left\{\gamma \in[0,1]: \overline{\chi(X)} \cap R_{\gamma} \neq \emptyset\right\} .
$$

That is, $\tilde{\mathcal{C}}_{\varphi, p}(\chi)=\alpha$ if and only if $\alpha_{2}=\alpha+\alpha_{1} \alpha / p$ is the straight line with the smallest slope among all the straight lines $\alpha_{2}=\gamma+\alpha_{1} \gamma / p$ touching the closure of $\chi(X)$ (see Figure 9).

Measures that are semicontinuous from below constructed in the above theorems have their 'dual' measures that are semicontinuous from above.

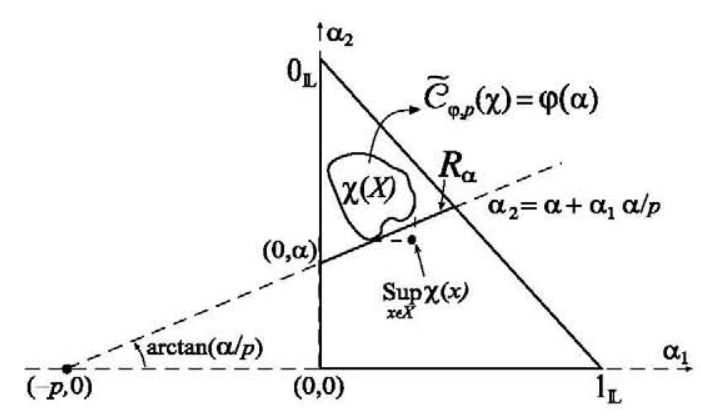

Figure 9. Geometrical interpretation of the measure constructed in Theorem 4.6. 
Theorem 4.7. Let $\varphi:[0,1] \rightarrow[0,1]$ be an order of automorphism, for all $p>0$, the function $\mathcal{C}^{\varphi, p}: \mathbb{L}^{X} \rightarrow[0,1]$ defined for each $\chi=(\mu, \nu) \in \mathbb{L}^{X}$ by (Figure 10)

$$
\mathcal{C}^{\varphi, p}(\chi)= \begin{cases}0 & \text { if } \chi \in \mathbb{L}_{0}^{X} \\ \varphi\left(\frac{p \operatorname{Sup}_{x \in X} \nu(x)}{p+\ln _{x \in X} \mu(x)}\right) & \text { if } \chi \in \mathbb{L}^{X} \backslash \mathbb{L}_{0}^{X}\end{cases}
$$

is a contradiction measure that is semicontinuous from above.

THEOREM 4.8 . Let $\varphi:[0,1] \rightarrow[0,1]$ be an order of automorphism, for all $p>0$, the function $\tilde{\mathcal{C}}^{\varphi, p}: \mathbb{L}^{X} \rightarrow[0,1]$ defined for each $\chi=(\mu, \nu) \in \mathbb{L}^{X}$ by (Figure 10)

$$
\tilde{\mathcal{C}}^{\varphi, p}(\chi)= \begin{cases}0 & \text { if } \chi \in \mathbb{L}_{0}^{X} \\ \operatorname{Sup}_{x \in X} \varphi\left(\frac{p \nu(x)}{p+\mu(x)}\right) & \text { if } \chi \in \mathbb{L}^{X} \backslash \mathbb{L}_{0}^{X}\end{cases}
$$

is a contradiction measure that is semicontinuous from above.

The measures $\mathcal{C}_{\varphi, p}$ and $\tilde{\mathcal{C}}_{\varphi, p}$ can be interpreted as a limit step of the measures $\mathcal{C}_{\varphi, p, Y}$ and $\tilde{\mathcal{C}}_{\varphi, p, Y}$, respectively, where the sequence $Y=\left\{y_{n}\right\}_{n \in \mathbb{N}}$ is replaced by the whole interval $[0,1]$, and the regions $R_{\gamma}$ are obtained by making $y_{n-1}$ match $y_{n}$. In the same way, the measures $\mathcal{C}^{\varphi, p}$ and $\tilde{\mathcal{C}}^{\varphi, p}$ can be considered as a limit step of the measures $\mathcal{C}^{\varphi, p, Y}$ and $\tilde{\mathcal{C}}^{\varphi, p, Y}$, respectively.

Now, let us establish a sufficient condition for $\mathcal{C}_{\varphi, p}$ and $\tilde{\mathcal{C}}_{\varphi, p}$ to take the same values on an A-IFS, and another one for $\mathcal{C}^{\varphi, p}$ and $\tilde{\mathcal{C}}^{\varphi, p}$ to be equal.

Proposition 4.9. Let $\varphi$ and $p$ be the same as in the previous theorems, and $\chi=(\mu, \nu) \in \mathbb{L}^{X}$, then the following holds.

(a) If $\{\chi(x)\}_{x \in X}$ is an upper semilattice, then $\mathcal{C}_{\varphi, p}(\chi)=\tilde{\mathcal{C}}_{\varphi, p}(\chi)$ holds.

(b) If $\{\chi(x)\}_{x \in X}$ is a lower semilattice, then $\mathcal{C}^{\varphi, p}(\chi)=\tilde{\mathcal{C}}^{\varphi, p}(\chi)$ holds.

Proof. To prove (a) we need to show that if $\{\chi(x)\}_{x \in X}$ is an upper semilattice, then

$$
\operatorname{Inf}_{x \in X} \frac{\nu(x)}{p+\mu(x)}=\frac{\operatorname{Inf}_{x \in X} \nu(x)}{p+\operatorname{Sup}_{x \in X} \mu(x)}
$$

and to prove (b) we need to show that if $\{\chi(x)\}_{x \in X}$ is a lower semilattice, then

$$
\operatorname{Sup}_{x \in X} \frac{\nu(x)}{p+\mu(x)}=\frac{\operatorname{Sup}_{x \in X} \nu(x)}{p+\operatorname{Inf}_{x \in X} \mu(x)} \text {. }
$$

The proof of the above two equalities is similar to the proof of equality (1) in Theorem 4.6.

Finally, let us see what kind of continuity the measures $\mathcal{C}_{\varphi, p}, \tilde{\mathcal{C}}_{\varphi, p}, \mathcal{C}^{\varphi, p}$, and $\tilde{\mathcal{C}}^{\varphi, p}$ do not satisfy.

Proposition 4.10. Let $\varphi:[0,1] \rightarrow[0,1]$ be an order of automorphism and let $p>0$. The following is satisfied.

(a) $\mathcal{C}_{\varphi, p}, \tilde{\mathcal{C}}_{\varphi, p} \notin \mathcal{C} \mathcal{M}_{c s c}\left(\mathbb{L}^{X}\right)$ and $\mathcal{C}^{\varphi, p}, \tilde{\mathcal{C}}^{\varphi, p} \notin \mathcal{C} \mathcal{M}^{c s c}\left(\mathbb{L}^{X}\right)$. 


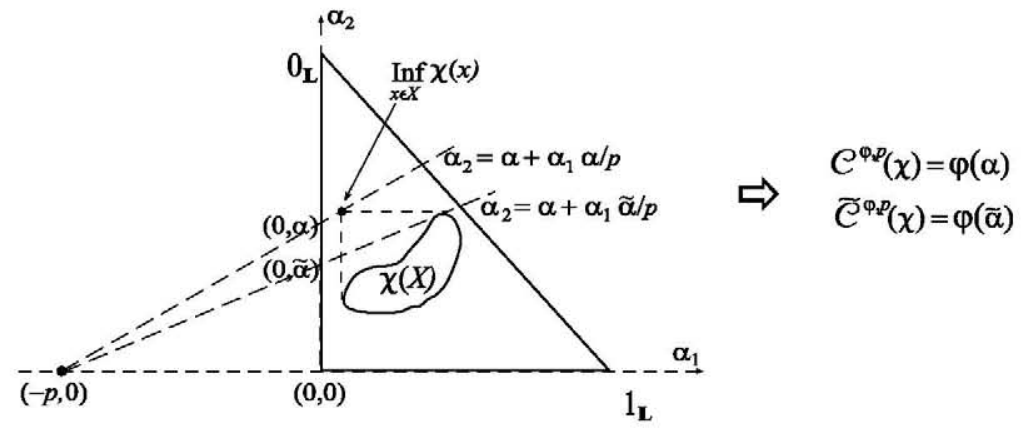

Figure 10. Geometrical interpretation of the measures constructed in Theorems 4.7 and 4.8.

(b) If $X$ is an infinite set, then $\mathcal{C}_{\varphi, p}, \tilde{\mathcal{C}}_{\varphi, p} \notin \mathcal{C} \mathcal{C}^{s c}\left(\mathbb{L}^{X}\right)$ (therefore $\mathcal{C}_{\varphi, p}, \tilde{\mathcal{C}}_{\varphi, p} \notin \mathcal{C} \mathcal{M}^{c s c}\left(\mathbb{L}^{X}\right)$ ) and $\mathcal{C}^{\varphi, p}, \tilde{\mathcal{C}}^{\varphi, p} \notin \mathcal{C} \mathcal{M}_{s c}\left(\mathbb{L}^{X}\right)$ (therefore $\mathcal{C}^{\varphi, p}, \tilde{\mathcal{C}}^{\varphi, p} \notin \mathcal{C} \mathcal{M}_{c s c}\left(\mathbb{L}^{X}\right)$ ).

Proof. (a) Let $\chi^{1}, \chi^{2} \in \mathbb{L}^{X}$ such that $\chi^{1}(x)=(0,1 / 2) \in R_{1 / 2}$ and

$$
\chi^{2}(x)=(p /(1+2 p),(1+p) /(1+2 p)) \in R_{1 / 2}
$$

for all $x \in X$, then $\operatorname{Sup}\left\{\chi^{1}, \chi^{2}\right\}(x)=(p /(1+2 p), 1 / 2) \in R_{(1+2 p) /(4(1+p))}$ and $\operatorname{Inf}\left\{\chi^{1}, \chi^{2}\right\}(x)=(0,(1+p) /(1+2 p)) \in R_{(1+p) /(1+2 p)}$ for all $x \in X$ as $1 / 2<(1+p) /$ $(1+2 p)$. Thus,

Therefore,

$$
\begin{aligned}
& \tilde{\mathcal{C}}_{\varphi, p}\left(\chi^{1}\right)=\mathcal{C}_{\varphi, p}\left(\chi^{1}\right)=\varphi\left(\frac{1}{2}\right) \quad \text { and } \quad \tilde{\mathcal{C}}_{\varphi, p}\left(\chi^{2}\right)=\mathcal{C}_{\varphi, p}\left(\chi^{2}\right)=\varphi\left(\frac{1}{2}\right), \\
& \tilde{\mathcal{C}}^{\varphi, p}\left(\chi^{1}\right)=\mathcal{C}^{\varphi, p}\left(\chi^{1}\right)=\varphi\left(\frac{1}{2}\right) \quad \text { and } \quad \tilde{\mathcal{C}}^{\varphi, p}\left(\chi^{2}\right)=\mathcal{C}^{\varphi, p}\left(\chi^{2}\right)=\varphi\left(\frac{1}{2}\right) .
\end{aligned}
$$

$$
\begin{gathered}
\operatorname{Inf}\left\{\tilde{\mathcal{C}}_{\varphi, p}\left(\chi^{1}\right), \tilde{\mathcal{C}}_{\varphi, p}\left(\chi^{2}\right)\right\}=\operatorname{Inf}\left\{\mathcal{C}_{\varphi, p}\left(\chi^{1}\right), \mathcal{C}_{\varphi, p}\left(\chi^{2}\right)\right\}=\varphi\left(\frac{1}{2}\right), \\
\operatorname{Sup}\left\{\tilde{\mathcal{C}}^{\varphi, p}\left(\chi^{1}\right), \tilde{\mathcal{C}}^{\varphi, p}\left(\chi^{2}\right)\right\}=\operatorname{Sup}\left\{\mathcal{C}^{\varphi, p}\left(\chi^{1}\right), \mathcal{C}^{\varphi, p}\left(\chi^{2}\right)\right\}=\varphi\left(\frac{1}{2}\right)
\end{gathered}
$$

and, nevertheless,

$$
\begin{gathered}
\tilde{\mathcal{C}}_{\varphi, p}\left(\operatorname{Sup}\left\{\chi^{1}, \chi^{2}\right\}\right)=\mathcal{C}_{\varphi, p}\left(\operatorname{Sup}\left\{\chi^{1}, \chi^{2}\right\}\right)=\varphi\left(\frac{1+2 p}{4(1+p)}\right)<\varphi\left(\frac{1}{2}\right), \\
\tilde{\mathcal{C}}^{\varphi, p}\left(\operatorname{Inf}\left\{\chi^{1}, \chi^{2}\right\}\right)=\mathcal{C}^{\varphi, p}\left(\operatorname{Inf}\left\{\chi^{1}, \chi^{2}\right\}\right)=\varphi\left(\frac{1+p}{1+2 p}\right)>\varphi\left(\frac{1}{2}\right) .
\end{gathered}
$$

(b) As in Proposition 3.3, again consider $\mathcal{P}_{F}(X)$ and let $\left\{\chi^{A}\right\}_{A \in \mathcal{P}_{F}(X)},\left\{\chi_{B}\right\}_{B \in \mathcal{P}_{F}(X)} \subset$ $\mathbb{L}^{X}$ be the families such that for each $A, B \in \mathcal{P}_{F}(X)$,

$$
\begin{aligned}
& \chi^{A}(x)= \begin{cases}0_{\mathbb{L}} & \text { if } x \in A \\
\left(0, \frac{1}{2}\right) & \text { if } x \notin A\end{cases} \\
& \chi_{B}(x)=\left\{\begin{array}{cc}
\left(0, \frac{1}{2}\right) & \text { if } x \in B \\
0_{\mathbb{L}} & \text { if } x \notin B
\end{array}\right.
\end{aligned}
$$


It holds that $\left\{\chi^{A}\right\}_{A \in \mathcal{P}_{F}(X)} \subset \mathbb{L}^{X} \backslash \mathbb{L}_{0}^{X}$ is a lower semilattice, and $\left\{\chi_{B}\right\}_{B \in \mathcal{P}_{F}(X)}$ is an upper semilattice, as if $\chi^{A_{1}}, \chi^{A_{2}} \in\left\{\chi^{A}\right\}_{A \in \mathcal{P}_{F}(X)}$, then $\operatorname{Inf}\left\{\chi^{A_{1}}, \chi^{A_{2}}\right\}=\chi^{A_{1} \cup A_{2}} \in\left\{\chi^{A}\right\}_{A \in \mathcal{P}_{F}(X)}$ and if $\chi_{B_{1}}, \chi_{B_{2}} \in\left\{\chi_{B}\right\}_{B \in \mathcal{P}_{F}(X)}$, then $\operatorname{Sup}\left\{\chi_{B_{1}}, \chi_{B_{2}}\right\}=\chi_{B_{1} \cup B_{2}} \in\left\{\chi_{B}\right\}_{B \in \mathcal{P}_{F}(X)}$. Furthermore, $\tilde{\mathcal{C}}_{\varphi, p}\left(\chi^{A}\right)=\mathcal{C}_{\varphi, p}\left(\chi^{A}\right)=\varphi(1 / 2)$ for all $\chi^{A} \in\left\{\chi^{A}\right\}_{A \in \mathcal{P}_{F}(X)}$ and $\tilde{\mathcal{C}}^{\varphi, p}\left(\chi_{B}\right)=\mathcal{C}^{\varphi, p}\left(\chi_{B}\right)=$ $\varphi(1)=1$ for all $\chi_{B} \in\left\{\chi_{B}\right\}_{B \in \mathcal{P}_{F}(X)}$, and so

$$
\begin{gathered}
\operatorname{Sup}_{A \in \mathcal{P}_{F}(X)} \tilde{\mathcal{C}}_{\varphi, p}\left(\chi^{A}\right)=\operatorname{Sup}_{A \in \mathcal{P}_{F}(X)} \mathcal{C}_{\varphi, p}\left(\chi^{A}\right)=\varphi\left(\frac{1}{2}\right)<1, \\
\operatorname{Inf}_{B \in \mathcal{P}_{F}(X)} \tilde{\mathcal{C}}^{\varphi, p}\left(\chi_{B}\right)=\operatorname{Inf}_{B \in \mathcal{P}_{F}(X)} \mathcal{C}^{\varphi, p}\left(\chi_{B}\right)=1 .
\end{gathered}
$$
thus,

However, $\operatorname{Inf}_{A \in \mathcal{P}_{F}(X)} \chi^{A}(x)=0_{\mathbb{L}}$ and $\operatorname{Sup}_{B \in \mathcal{P}_{F}(X)} \chi_{B}(x)=(0,1 / 2)$ for all $x \in X$, and

$$
\begin{gathered}
\tilde{\mathcal{C}}_{\varphi, p}\left(\operatorname{Inf}_{A \in \mathcal{P}_{F}(X)} \chi^{A}\right)=\mathcal{C}_{\varphi, p}\left(\operatorname{Inf}_{A \in \mathcal{P}_{F}(X)} \chi^{A}\right)=1, \\
\tilde{\mathcal{C}}^{\varphi, p}\left(\operatorname{Sup}_{B \in \mathcal{P}_{F}(X)} \chi_{B}\right)=\mathcal{C}^{\varphi, p}\left(\operatorname{Sup}_{B \in \mathcal{P}_{F}(X)} \chi_{B}\right)=\varphi\left(\frac{1}{2}\right)<1 .
\end{gathered}
$$

\section{Conclusions}

In this work, we have tackled the problem of obtaining new families of contradiction measures in the framework of A-IFSs. In previous papers, we proposed different types of such measures, depending on the continuity properties they satisfy, and gave some examples of such types of contradiction measures. But the challenge of obtaining a lot of measures systematically remained open.

On the one hand, we cited in remark 2 and Section 3 examples that were given in a previous paper. These examples defined contradiction measures that take discrete values depending on specific regions, these regions being a partition of the lattice $\mathbb{L}$. Based on these examples, we have given general methods for building contradiction measures in the following manner: by generalizing the determination of the regions of $\mathbb{L}$ and using isomorphisms and decreasing functions.

On the other hand, we have introduced new methods of constructing contradiction measures. These take all the values of the interval $[0,1]$. Furthermore, these measures can be interpreted as a limit step of the above measures that took discrete values.

The key to all these constructions is to determinate the regions of $\mathbb{L}$ in order to define the values of the contradiction measures. In the case of completely semicontinuous measures, those regions are semilattices.

\section{Acknowledgement}

This paper is partially supported by Ministerio de Ciencia e Innovación (DGI, Spain) under project TIN2008-06890-C02-01 and by UPM-CAM.

\section{Notes}

1. Email: ecastineira@fi.upm.es

2. Email: scubillo@fi.upm.es 


\section{Notes on contributors}

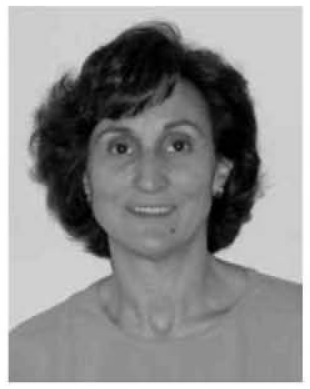

Elena Castiñeira Holgado was born in Asturias (Spain). She received her bachelors degree in Mathematics from the Complutense University of Madrid in 1985, and her PhD in Computer Science from the Technical University of Madrid in 1994, with research on fractals. She has been working on non-classical logics, and, especially, fuzzy logic since 1997.

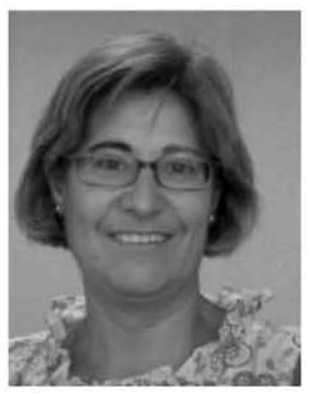

Carmen Torres Blanc was born in Albacete (Spain). She received her bachelors degree in Mathematics from the Complutense University of Madrid in 1985, and her $\mathrm{PhD}$ in Mathematic Science from the Technical University of Madrid in 2000 , with research on digital control systems. She has been working on fuzzy logic and Atanassov intuitionistic fuzzy sets since 2004 .

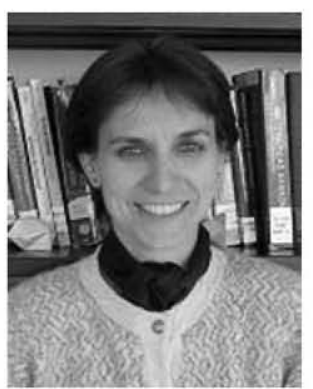

Susana Cubillo Villanueva was born in Madrid (Spain). She received her bachelors degree in Mathematics from the Complutense University of Madrid in 1981, and her PhD in Computer Science from the Technical University of Madrid in 1993, with a thesis on logic and fuzzy conditionals. She has written numerous research articles on fuzzy logic and nonmonotonic reasoning since 1990 .

\section{References}

Atanassov, K.T., 1986. Intuitionistic fuzzy sets. Fuzzy sets and systems, 20, 87-96.

Atanassov, K.T., 1999. Intuitionistic fuzzy sets: theory and applications. Heidelberg: PhysicaVerlag.

Birkhoff, G., 1940. Lattice theory. American Mathematical Society.

Blyth, T., 2005. Lattices and ordered algebraic structures. Springer-Verlag.

Bustince, H., Kacprzyk, J., and Mohedano, V., 2000. Intuitionistic fuzzy generators - application to intuitionistic fuzzy complementation. Fuzzy sets and systems, 114, 485-504.

Castiñeira, E.E. and Cubillo, S., 2009. Measures of self-contradiction in Atanassov's fuzzy sets: an axiomatic model. International journal of intelligent systems, 24, 863-888.

Castiñeira, E.E., Cubillo, S., and Bellido, S., 2002. Degrees of contradiction in fuzzy sets theory. In: Proceedings of the IX conference of information processing and management of uncertainty in knowledge-based systems (IPMU). Annecy, France, 171-176.

Castiñeira, E.E., Cubillo, S., and Torres-Blanc, C., 2006. Searching degrees of self-contradiction in Atanassov's fuzzy sets. Mathware and soft-computing, 13 (3), 139-156.

Cubillo, S. and Castiñeira, E.E., 2004. Contradiction in intuitionistic fuzzy sets. In: Proceedings of the IPMU. Perugia, Italy, 2180-2186.

Cubillo, S. and Castiñeira, E.E., 2005. Measuring contradiction in fuzzy logic. International journal of general systems, 34 (1), 39-59. 
Deschrijver, G., Cornelis, C., and Kerre, E., 2002. Intuitionistic fuzzy connectives revisited. In: Proceedings of the IX conference of information processing and management of uncertainty in knowledge-based systems (IPMU). Annecy, France, 1839-1844.

Deschrijver, G., Cornelis, C., and Kerre, E., 2004. On the representation of intuitionistic fuzzy t-norms and t-conorms. IEEE transactions on fuzzy systems, 12 (1), 45-61.

Goguen, J.A., 1967. L-fuzzy sets. Journal of mathematical analysis and applications, 18 (1), $623-668$.

Piasecki, K., 1985. Probability of fuzzy events defined as denumerable additivity measure. Fuzzy sets and systems, 17, 271-284.

Sugeno, M., 1974. Theory of fuzzy integrals and its applications. Thesis (PhD). Tokyo Institute of Technology, Japan.

Trillas, E., Alsina, C., and Jacas, J., 1999. On contradiction in fuzzy logic. Soft computing, 3 (4), 197-199.

Trillas, E., Alsina, C., and Pradera, A., 2002. Searching for the roots of non-contradiction and excluded-middle. International journal of general systems, 31 (5), 499-513.

Trillas, E. and Cubillo, S., 1999. On non-contradictory input/output couples in Zadeh's CRI. In: Proceedings of the NAFIPS. New York, 28-32.

Yager, R., 1980. On a general class of fuzzy connectives. Fuzzy sets and systems, 4, 235-242. 